\title{
9. A HIGH RESOLUTION LATE MIOCENE-PLIOCENE DIATOM BIOSTRATIGRAPHY FOR THE EASTERN EQUATORIAL PACIFIC ${ }^{1}$
}

\author{
Jack G. Baldauf, U.S. Geological Survey, Menlo Park, and Department of Paleontology, \\ University of California at Berkeley²
}

\begin{abstract}
Quantitative procedures are used here for DSDP Sites 572, 573, and 503 to refine the stratigraphic resolution for upper Neogene sediments of the eastern equatorial Pacific Ocean. As a result, six new diatom events are defined for the stratigraphic interval 6.2 to $1.5 \mathrm{Ma}$. The carbonate minimums $5 \mathrm{e}, \mathrm{Gu} 3$, and $\mathrm{M} 21$, as well as the carbonate maximums $5 \mathrm{f}$ and $5 \mathrm{~d}$, are correlated to diatom datums and are useful in refining the stratigraphic resolution of the upper Neogene. In addition to allowing stratigraphic refinement, quantitative procedures aid in determining paleoceanographic conditions. The greater abundance of Coscinodiscus nodulifer at Site 503 as compared with Sites 572 and 573 suggests that this species is associated with equatorial upwelling. The greater abundance of Thalassionema nitzschioides at Site 572 suggests that this species is associated with the Equatorial Undercurrent. Furthermore, the gradual decline in the abundance of $T$. nitzschioides between 4.2 and $3.7 \mathrm{Ma}$ at Sites 572 and 503 may have resulted from restriction in surface-water communication between the Atlantic and Pacific Oceans as a result of the emergence of the Isthmus of Panama.
\end{abstract}

\section{INTRODUCTION}

Numerous biostratigraphic studies of the equatorial Pacific (in particular, Burckle, 1969, 1972, 1977, 1978; Barron, in press) have resulted in the development and adoption of a middle Miocene-Holocene low-latitude diatom zonation. This zonal scheme, defined principally by Burckle $(1972,1977)$, is based on biostratigraphic events. With the exception of one event, these events are based on the first or last occurrences of selected, easily recognizable species that have a consistent stratigraphic range and a wide geographic distribution. The single exception is the evolutionary transition of Rhizosolenia praebergonii to $R$. praebergonii var. robusta, which is a stratigraphically useful event. These events have been correlated with magnetostratigraphy by Burckle $(1972,1977$, 1978), Burckle and Trainer (1979), and Barron et al. (in press).

Figure 1 presents the diatom datums defined (previously and in this paper) for the interval 6.5 to $1.5 \mathrm{Ma}$ and their correlation to the paleomagnetic scale of Berggren et al. (in press). The occurrence of 25 datums within this 5-m.y. interval (Column 1, Fig. 1) indicates that the average temporal resolution is 1 datum per $0.2 \mathrm{~m}$.y. However, this resolution is not uniform because the datums are generally concentrated within specific time intervals. For example, only two datums occur within the Gauss paleomagnetic chron, whereas six datums are defined for an equivalent duration of time within the older part of the Matuyama paleomagnetic chron.

The use of changes in relative abundances in addition to first and last occurrences results in an increase in the number of definable datums, thereby increasing the avail-

\footnotetext{
${ }^{1}$ Mayer, L., Theyer, F., et al., Init. Repts. DSDP, 85: Washington (U.S. Govt. Printing Office). 77843.
}

able temporal resolution (Column 2, Fig. 1). Quantitative procedures using diatoms were first made popular by Burckle (1977), who defined two diatom events for the Quaternary. These Quaternary datums are based on the progressive upward increase in abundance of Thalassiosira oestrupii from 5 to $20 \%$ of the overall diatom assemblage and by a distinct acme in Roperia tesselata var. ovata.

Burckle and Trainer (1979) subsequently defined six additional datums for the interval including the upper Gilbert Chron to slightly above the Olduvai Subchron within the Matuyama Chron. These datums defined by Burckle and Trainer are based on the disappearance and reappearance of $T$. convexa and Coscinodiscus nodulifer var. cyclopus in Core V28-179 from the equatorial Pacific.

To further refine the stratigraphic resolution of the middle and upper Neogene, the quantitative biostratigraphic approach used by Burckle and Trainer (1979) is here extended to older floras through the lower Pliocene and uppermost Miocene sequences on the basis of samples recovered from three sites located within the eastern equatorial Pacific (Fig. 2).

At least three factors should be taken into consideration if stratigraphic correlations based on fluctuations in fossil abundance are to be valid. First, species abundance is influenced by environment and will vary with fluctuations in climate, the migration of water masses, nutrient limitations, and biological factors (i.e., predation and competition). For a correlation between abundance peaks to be valid, the main environmental conditions that affect diatom abundance must be similar in the regions of interest. Second, abundance fluctuations may be cyclical, and the frequency of the fluctuations may be unknown, so a sampling program may be insufficient to document all the fluctuations in the abundance of a given species. To be valid, correlated abundance peaks must be coeval, and to ensure that they are re- 


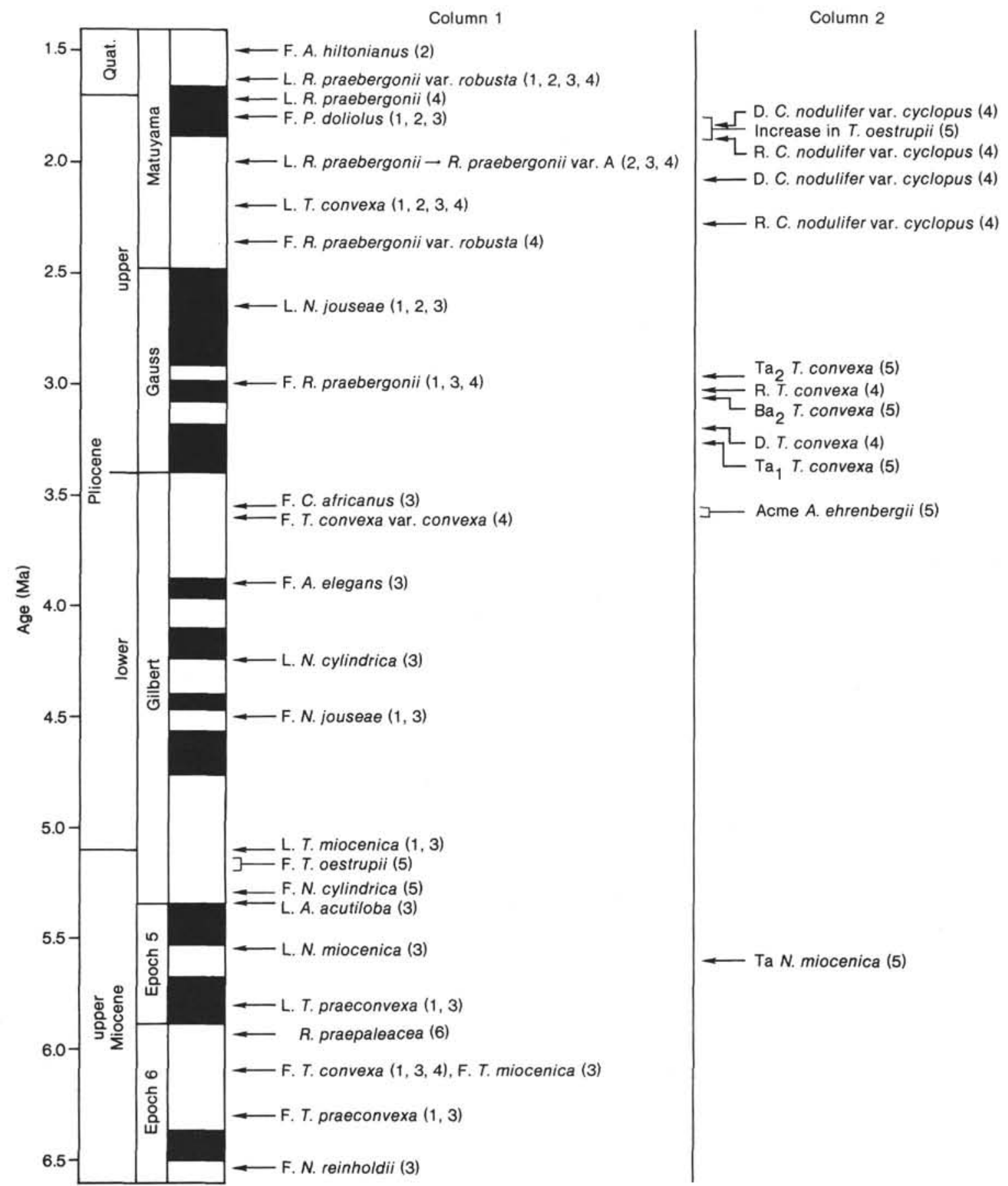

Figure 1. Correlation of useful diatom datums to the paleomagnetic scale of Berggren et al. (in press). Column 1 consists of datums defined on the first or last occurrence of specific species (with the exception of the evolutionary transition of $R$. praebergonii). Column 2 consists of diatom datums resulting from quantitative analysis. $\mathrm{Ta}=$ top of abundance interval; $\mathrm{D}=$ disappearance; $\mathrm{Ba}=$ bottom of abundance interval; $\mathrm{R}=$ recurrence. References: (1) Burckle, 1972; (2) Burckle, 1977; (3) Burckle, 1978; (4) Burckle and Trainer, 1979; (5) this paper; (6) Barron et al., in press.

quires a large number of stratigraphically closely spaced samples and an independent stratigraphic control (isotopes, paleomagnetism, or other fossil groups). Third, it is necessary to know the stratigraphic and counting precision to ensure that correlated peaks in abundance are coeval.

These constraints limit the usefulness of fluctuations in abundance for purposes of stratigraphic correlation; the results of this study are preliminary. The author in- tends to use these results to determine major fluctuations of a species within specific stratigraphic intervals and to study these intervals further with more closely spaced samples from the same geographic region.

Sites 573, 572, and 503 form a west-east transect from the central Pacific (Site 573), characterized by low surface-water productivity, to the Guatemala basin region (Site 503 ), characterized by high surface-water productivity (Fig. 2). The intermediate site (572) is located at 


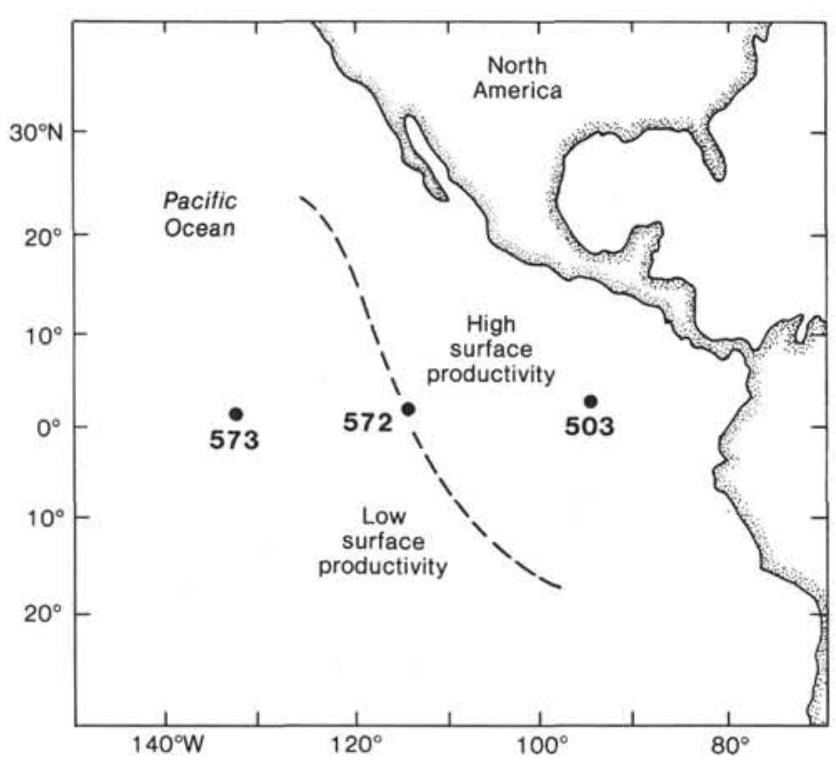

Figure 2. Geographic locations of Sites 573, 572 (Leg 85), and 503 (Leg 68). Dashed line represents inferred boundary between areas of high and low productivity.

the westernmost fringe of the high productivity region. Because of the geographic position of these sites, this study provides information on the paleoceanography of the region in addition to a refinement of the stratigraphy.

\section{METHODOLOGY}

At least one sample was examined for each 0.1-m.y. period within the time interval 6.2 to $1.5 \mathrm{Ma}$, except when recovery was poor or when hiatuses were present. The length of each $0.1-\mathrm{m} . \mathrm{y}$. period was extrapolated from sediment accumulation rate curves derived from paleomagnetic and paleontologic data. When available, more than one sample was examined for each 0.1-m.y. period. For the interval younger than $3.5 \mathrm{Ma}$, the sediment accumulation curve is based on both paleomagnetic and biostratigraphic data; age estimates are carried to two digits after the decimal. For the interval older than $3.5 \mathrm{Ma}$, the sediment accumulation curve is based solely on biostratigraphic data. Age estimates are, therefore, carried to one digit after the decimal. The precision is greatest when the stratigraphy is based on biostratigraphic, paleomagnetic, and isotopic data. Use of biostratigraphic data alone for the interval older than $3.5 \mathrm{Ma}$ assumes isochroneity of biostratigraphic datums, an assumption that cannot be tested without independent (e.g., paleomagnetic or isotopic) stratigraphies. Further studies are required to determine if a $0.1-$ m.y. age difference between two abundance peaks in different cores is significant for this time interval.

Samples were processed and strewn slides were prepared following the procedures outlined in Baldauf (1985). Strewn slides were examined in their entirety. The first 300 diatoms observed at $\times 1250$ were tabulated. The remaining portion of the slide was examined at $\times 500$ for additional stratigraphically useful species. The counting procedures of Schrader and Gersonde (1978) were used. The age assigned to each sample (see Tables 1 to 3 ) is extrapolated from the sedimentation accumulation curves shown in Figure 3. For taxonomic descriptions and figures of species discussed in this report, the reader is referred to Barron (this volume) and Baldauf (1985).

\section{RESULTS}

Tables 1 through 3 show the relative frequency of species recorded at each site. The first or last occurrences of stratigraphically useful species are used in determining the sediment accumulation rate curves (Fig. 3). In addition, the paleomagnetic results of Hailwood and Rees (1982, Site 503) and Weinreich and Theyer (this volume, Site 573) are used where applicable.

With the exception of two hiatuses having durations of about 0.5 m.y., a continuous sedimentary sequence is present at all sites. However, no sediment was recovered from Cores 503A-25, -26 , and -28; thus, with the exception of Sample 503A-27,CC, no samples in the stratigraphic interval representing 4.8 to $4.4 \mathrm{Ma}$ were examined at Site 503. The oldest hiatus occurs within the lower Pliocene at Site 573, approximately between the last occurrence of Thalassiosira miocenica and the first occurrence of Nitzschia jouseae. This hiatus occurs from approximately 5.1 to $4.5 \mathrm{Ma}$, which suggests that it corresponds to the NH7 hiatus of Barron and Keller (1982) and Keller and Barron (1983). The NH7 hiatus extends from 5.2 to $4.7 \mathrm{Ma}$, an interval noted by Barron and Keller (1982) as corresponding to a period of diatom dissolution in the eastern equatorial Pacific. The early Pliocene hiatus observed at Site 573 is also recognized by Barron (this volume) at Site 575. A stratigraphic compression of this same interval occurs at Site 574 and could have resulted from a reduced sedimentation rate rather than from erosion or dissolution. At Site 575 the early Pliocene hiatus is expanded and extends upward to the first occurrence of Rhizosolenia praebergonii (3.0 $\mathrm{Ma}$ ). The second hiatus is late Pliocene in age and is observed at Site 572. This hiatus occurs within the interval between the last occurrence of $T$. convexa Group at 2.2 $\mathrm{Ma}$ and the first occurrence of Pseudoeunotia doliolus at $1.8 \mathrm{Ma}$.

Although sedimentation rates are slightly different at each site (see Fig. 3), similarities are observed between sites. First, an abrupt change in the sedimentation rate from $73.0 \mathrm{~m} / \mathrm{m}$.y. to $39.5 \mathrm{~m} / \mathrm{m}$.y. occurs at Site 572 $(\sim 5.6 \mathrm{Ma})$ and at Site $503(\sim 5.8 \mathrm{Ma})$. Second, a change in the sedimentation rate from $39.5 \mathrm{~m} / \mathrm{m} . \mathrm{y}$. to $15.0 \mathrm{~m} /$ m.y. also occurs at Site $572(\sim 3.6 \mathrm{Ma})$ and at Site 573 ( $\sim 3.9 \mathrm{Ma})$.

Figures 4 through 12 present the fluctuation in the relative frequency of selected species. As a result of this quantitative analysis, six datums are defined for the interval 6.2 to $1.5 \mathrm{Ma}$ on the basis of acmes of individual species.

\section{Peak in the Abundance of Nitzschia miocenica}

Figures 4 to 6 show the relative frequency of Nitzschia miocenica from the samples examined. At both Sites 572 and 503 (Figs. 4 and 5), N. miocenica ordinarily composes 10 to $15 \%$ of the overall diatom assemblage throughout its stratigraphic range. However, a decline in abundance from approximately $15 \%$ to less than $5 \%$ is observed near the top of the stratigraphic range of $N$. miocenica. Approximately $0.2 \mathrm{~m} . \mathrm{y}$. above the start of this decline $N$. miocenica becomes extinct. This peak in abundance has an extrapolated age of $5.6 \mathrm{Ma}$ at both Sites 572 and 503.

At Site 573 (Fig. 6) a similar decline in abundance from approximately $20 \%$ to less than $5 \%$ is observed. The peak in abundance, however, occurs at approximately $5.8 \mathrm{Ma}$, which differs from the extrapolated age. This 
Table 1. Relative frequency of species encountered during examination of samples from Site 572 .

\begin{tabular}{|c|c|c|c|c|c|c|c|c|c|c|c|c|c|c|c|c|c|c|c|c|c|c|}
\hline \multicolumn{2}{|c|}{$\begin{array}{l}\text { Diatom } \\
\text { zonation }\end{array}$} & $\begin{array}{c}\text { Sample } \\
\text { interval } \\
(\mathrm{cm})\end{array}$ & $\begin{array}{l}\text { Depth } \\
\text { (m) }\end{array}$ & 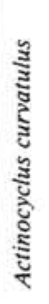 & 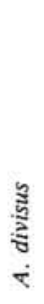 & ¿ัँ & 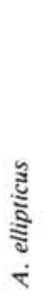 & 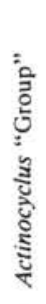 & 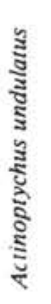 & 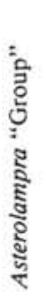 & 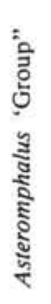 & 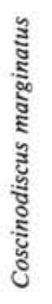 & 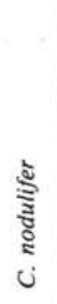 & 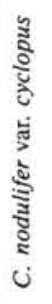 & 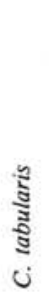 & 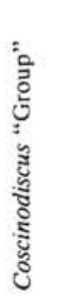 & 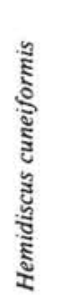 & 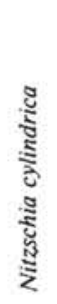 & 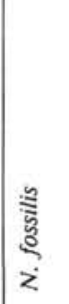 & 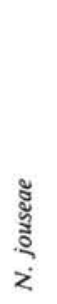 & $\begin{array}{l}\text { ह } \\
\text { है } \\
z\end{array}$ & 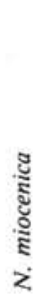 \\
\hline \multicolumn{2}{|c|}{$\begin{array}{l}\text { Pseudoeunotia } \\
\text { doliolus }\end{array}$} & $\begin{array}{l}\text { Hole 572A } \\
\begin{array}{l}3-6,68-69 \\
3, C C \\
4-1,68-69 \\
4-2,66-67 \\
4-3,66-67 \\
\end{array}\end{array}$ & $\begin{array}{l}27.2 \\
28.6 \\
29.3 \\
30.8 \\
32.3 \\
\end{array}$ & $\begin{array}{l}\overline{1} \\
\overline{-} \\
-\end{array}$ & $\begin{array}{l}+ \\
- \\
+ \\
+ \\
1\end{array}$ & $\begin{array}{l}+ \\
- \\
- \\
1 \\
2\end{array}$ & $\begin{array}{l}- \\
- \\
\overline{1} \\
2\end{array}$ & $\begin{array}{l}\overline{1} \\
\overline{-} \\
\overline{-}\end{array}$ & $\begin{array}{l}- \\
- \\
\overline{-} \\
-\end{array}$ & $\begin{array}{l}\overline{1} \\
+ \\
- \\
-\end{array}$ & $\begin{array}{l}2 \\
4 \\
2 \\
4 \\
5 \\
\end{array}$ & $\begin{array}{l}1 \\
1 \\
- \\
1\end{array}$ & $\begin{array}{l}5 \\
5 \\
2 \\
8 \\
6 \\
\end{array}$ & $\begin{array}{l}+ \\
1 \\
- \\
- \\
2\end{array}$ & $\begin{array}{l}4 \\
1 \\
3 \\
4 \\
4 \\
\end{array}$ & $\begin{array}{l}1 \\
- \\
1 \\
1\end{array}$ & $\begin{array}{l}4 \\
3 \\
3 \\
3 \\
2 \\
\end{array}$ & $\begin{array}{l}- \\
- \\
- \\
- \\
-\end{array}$ & \begin{tabular}{|r}
9 \\
5 \\
11 \\
14 \\
7 \\
\end{tabular} & $\begin{array}{l}\overline{-} \\
\overline{-} \\
\overline{-} \\
\end{array}$ & $\begin{array}{l}\overline{3} \\
1 \\
2 \\
1 \\
\end{array}$ & $\begin{array}{l}\overline{-} \\
\overline{-} \\
\overline{-}\end{array}$ \\
\hline $\begin{array}{l}\text { Rhizosolenia } \\
\text { praebergonii }\end{array}$ & c & $\begin{array}{l}4-4,66-67 \\
4-5,66-67 \\
4, C C \\
5-1,66-67 \\
5-2,66-67 \\
5-3,66-67 \\
5-4,66-67 \\
5-5,66-67 \\
5-6,66-67 \\
5, C C \\
6-1,66-67 \\
6-2,66-67\end{array}$ & $\begin{array}{l}33.8 \\
35.3 \\
36.2 \\
37.0 \\
38.5 \\
40.0 \\
41.5 \\
43.0 \\
44.5 \\
45.8 \\
46.6 \\
48.1 \\
\end{array}$ & $\begin{array}{l}\overline{-} \\
1 \\
+ \\
\overline{1} \\
+ \\
- \\
- \\
+ \\
- \\
-\end{array}$ & $\begin{array}{l}1 \\
+ \\
- \\
1 \\
+ \\
+ \\
1 \\
- \\
+ \\
\overline{1} \\
-\end{array}$ & $\begin{array}{l}-\overline{1} \\
1 \\
1 \\
+ \\
1 \\
1 \\
1 \\
1 \\
+ \\
- \\
-\end{array}$ & $\begin{array}{l}- \\
1 \\
1 \\
1 \\
1 \\
1 \\
1 \\
1 \\
5 \\
1 \\
+ \\
3\end{array}$ & $\begin{array}{l}+ \\
+ \\
+ \\
- \\
+ \\
+ \\
- \\
\overline{1} \\
- \\
-\end{array}$ & $\begin{array}{l}- \\
\overline{-} \\
\overline{-} \\
\overline{-} \\
\overline{-} \\
\overline{-} \\
\overline{-} \\
-\end{array}$ & $\begin{array}{l}\overline{1} \\
- \\
- \\
+ \\
- \\
- \\
+ \\
- \\
- \\
- \\
-\end{array}$ & $\begin{array}{l}\mathrm{e} \\
5 \\
2 \\
4 \\
3 \\
2 \\
3 \\
5 \\
1 \\
2 \\
3 \\
1\end{array}$ & $\begin{array}{l}+ \\
- \\
1 \\
+ \\
1 \\
+ \\
+ \\
+ \\
+ \\
1 \\
- \\
-\end{array}$ & $\begin{array}{r}5 \\
12 \\
3 \\
6 \\
12 \\
5 \\
5 \\
7 \\
11 \\
4 \\
8 \\
8\end{array}$ & $\begin{array}{l}1 \\
3 \\
- \\
- \\
2 \\
- \\
- \\
- \\
+ \\
- \\
1 \\
-\end{array}$ & $\begin{array}{l}2 \\
2 \\
2 \\
6 \\
3 \\
3 \\
3 \\
5 \\
3 \\
1 \\
3 \\
2\end{array}$ & $\begin{array}{l}1 \\
1 \\
1 \\
+ \\
+ \\
- \\
- \\
1 \\
1 \\
+ \\
1 \\
-\end{array}$ & $\begin{array}{l}3 \\
6 \\
3 \\
2 \\
4 \\
4 \\
1 \\
4 \\
7 \\
4 \\
3 \\
1\end{array}$ & $\begin{array}{l}- \\
- \\
- \\
- \\
- \\
\overline{-} \\
- \\
- \\
- \\
- \\
-\end{array}$ & $\begin{array}{r}18 \\
8 \\
7 \\
12 \\
9 \\
10 \\
7 \\
8 \\
10 \\
4 \\
6 \\
3\end{array}$ & $\begin{array}{l}- \\
- \\
- \\
- \\
- \\
- \\
2 \\
3 \\
9 \\
6 \\
5 \\
7\end{array}$ & $\begin{array}{l}1 \\
+ \\
4 \\
1 \\
1 \\
2 \\
1 \\
1 \\
2 \\
3 \\
+ \\
-\end{array}$ & $\begin{array}{l}- \\
\overline{-} \\
- \\
- \\
\overline{-} \\
\overline{-} \\
- \\
- \\
-\end{array}$ \\
\hline \multicolumn{2}{|c|}{$\begin{array}{l}\text { Nitzschia } \\
\text { jouseae }\end{array}$} & $\begin{array}{l}6-3,66-67 \\
6-4,66-67 \\
6-5,66-67 \\
6-6,66-67 \\
6, \mathrm{CC} \\
7-1,66-67 \\
7-2,66-67 \\
7-3,66-67 \\
7-4,66-67 \\
7-5,66-67 \\
7-6,66-67 \\
7, \mathrm{CC} \\
8-3,66-67 \\
8, \mathrm{CC} \\
9-3,66-67 \\
9, \mathrm{CC} \\
10-3,66-67 \\
10, \mathrm{CC}\end{array}$ & $\begin{array}{l}49.6 \\
51.1 \\
52.6 \\
54.1 \\
55.1 \\
55.9 \\
57.4 \\
58.9 \\
60.4 \\
61.9 \\
63.4 \\
64.0 \\
67.8 \\
72.3 \\
76.0 \\
81.7 \\
85.5 \\
91.4 \\
\end{array}$ & $\begin{array}{l}1 \\
1 \\
- \\
- \\
- \\
- \\
+ \\
- \\
- \\
1 \\
+ \\
1 \\
1 \\
1 \\
1 \\
- \\
+\end{array}$ & $\begin{array}{l}\overline{1} \\
+ \\
- \\
- \\
\overline{-} \\
+ \\
- \\
\overline{1} \\
\overline{-} \\
- \\
- \\
\overline{1} \\
\overline{-} \\
\overline{-} \\
-\end{array}$ & $\begin{array}{l}+ \\
- \\
+ \\
1 \\
+ \\
3 \\
14 \\
+ \\
3 \\
- \\
+ \\
1 \\
1 \\
- \\
- \\
- \\
+ \\
-\end{array}$ & $\begin{array}{l}5 \\
2 \\
1 \\
1 \\
1 \\
1 \\
2 \\
3 \\
4 \\
3 \\
7 \\
3 \\
3 \\
2 \\
3 \\
3 \\
1 \\
- \\
\end{array}$ & $\begin{array}{l}\overline{-} \\
\overline{+} \\
- \\
+ \\
+ \\
+ \\
- \\
- \\
- \\
+ \\
- \\
- \\
+ \\
- \\
- \\
- \\
-\end{array}$ & $\begin{array}{l}- \\
\overline{-} \\
- \\
- \\
\overline{+} \\
- \\
- \\
\overline{1} \\
4 \\
4 \\
\overline{1} \\
- \\
+ \\
- \\
-\end{array}$ & $\begin{array}{l}- \\
\overline{-} \\
- \\
- \\
- \\
- \\
- \\
\overline{1} \\
1 \\
- \\
- \\
- \\
- \\
- \\
+ \\
+\end{array}$ & $\begin{array}{l}4 \\
1 \\
1 \\
1 \\
1 \\
1 \\
1 \\
2 \\
6 \\
5 \\
3 \\
1 \\
1 \\
1 \\
1 \\
1 \\
+ \\
+ \\
+\end{array}$ & $\begin{array}{l}1 \\
+ \\
+ \\
- \\
1 \\
- \\
+ \\
+ \\
+ \\
- \\
1 \\
+ \\
- \\
1 \\
1 \\
+ \\
-\end{array}$ & $\begin{array}{r}12 \\
2 \\
5 \\
5 \\
6 \\
10 \\
11 \\
11 \\
10 \\
9 \\
7 \\
4 \\
2 \\
1 \\
- \\
5 \\
6 \\
1\end{array}$ & $\begin{array}{l}1 \\
1 \\
- \\
+ \\
- \\
+ \\
1 \\
1 \\
+ \\
2 \\
1 \\
- \\
1 \\
- \\
- \\
2 \\
- \\
-\end{array}$ & $\begin{array}{l}5 \\
1 \\
6 \\
6 \\
1 \\
2 \\
2 \\
1 \\
1 \\
3 \\
2 \\
2 \\
1 \\
- \\
- \\
+ \\
1 \\
- \\
\end{array}$ & $\begin{array}{l}1 \\
+ \\
- \\
1 \\
+ \\
- \\
1 \\
1 \\
+ \\
1 \\
+ \\
+ \\
+ \\
- \\
+ \\
+ \\
+ \\
-\end{array}$ & $\begin{array}{l}9 \\
2 \\
3 \\
- \\
3 \\
2 \\
4 \\
1 \\
3 \\
2 \\
2 \\
+ \\
\\
2 \\
1 \\
1 \\
2 \\
-\end{array}$ & $\begin{array}{l}- \\
- \\
- \\
- \\
- \\
- \\
- \\
- \\
- \\
- \\
- \\
- \\
- \\
- \\
- \\
- \\
3 \\
1\end{array}$ & $\begin{array}{r}7 \\
4 \\
2 \\
4 \\
3 \\
2 \\
3 \\
2 \\
4 \\
2 \\
8 \\
5 \\
13 \\
9 \\
7 \\
4 \\
1 \\
4 \\
\end{array}$ & $\begin{array}{r}4 \\
10 \\
6 \\
11 \\
8 \\
5 \\
13 \\
10 \\
10 \\
13 \\
14 \\
8 \\
10 \\
6 \\
5 \\
4 \\
8 \\
3\end{array}$ & $\begin{array}{l}2 \\
- \\
- \\
- \\
1 \\
+ \\
1 \\
+ \\
+ \\
1 \\
3 \\
1 \\
1 \\
1 \\
1 \\
+ \\
- \\
-\end{array}$ & $\begin{array}{l}- \\
\overline{-} \\
\overline{-} \\
\overline{-} \\
\overline{-} \\
\overline{-} \\
\overline{-} \\
\overline{-} \\
\overline{-} \\
\overline{-} \\
\overline{-}\end{array}$ \\
\hline \multirow[t]{2}{*}{$\begin{array}{l}\text { Thalassiosira } \\
\text { convexa }\end{array}$} & b & $\begin{array}{l}11-3,67-68 \\
11, \mathrm{CC} \\
12, \mathrm{CC} \\
13-3,66-67 \\
13, \mathrm{CC} \\
14, \mathrm{CC} \\
15-3,68-69 \\
15, \mathrm{CC} \\
16-3,66-67 \\
16, \mathrm{CC} \\
17, \mathrm{CC}\end{array}$ & \begin{tabular}{r|}
95.1 \\
100.6 \\
109.6 \\
113.6 \\
119.1 \\
128.2 \\
132.0 \\
136.7 \\
140.8 \\
145.2 \\
154.1
\end{tabular} & $\begin{array}{l}- \\
\overline{-} \\
\overline{1} \\
+ \\
+ \\
\overline{-} \\
\overline{-} \\
\overline{-} \\
\overline{-}\end{array}$ & $\begin{array}{l}+ \\
1 \\
+ \\
- \\
+ \\
1 \\
- \\
1 \\
- \\
1 \\
1\end{array}$ & $\begin{array}{l}+ \\
+ \\
+ \\
+ \\
1 \\
- \\
1 \\
+ \\
1 \\
-\end{array}$ & $\begin{array}{l}2 \\
2 \\
+ \\
+ \\
+ \\
1 \\
3 \\
1 \\
1 \\
+ \\
2\end{array}$ & $\begin{array}{l}- \\
\overline{-} \\
\overline{-} \\
\overline{2} \\
+ \\
+ \\
\overline{1} \\
\overline{1} \\
-\end{array}$ & $\begin{array}{l}\overline{-} \\
\overline{-} \\
\overline{-} \\
\overline{-} \\
\overline{+} \\
\overline{-} \\
-\end{array}$ & $\begin{array}{l}+ \\
- \\
- \\
+ \\
1 \\
+ \\
1 \\
- \\
\overline{1} \\
-\end{array}$ & $\begin{array}{l}1 \\
1 \\
+ \\
+ \\
2 \\
1 \\
-1 \\
1 \\
-1 \\
1 \\
1\end{array}$ & $\begin{array}{l}- \\
+ \\
- \\
- \\
+ \\
+ \\
+ \\
\\
+ \\
+\end{array}$ & $\begin{array}{r}3 \\
3 \\
1 \\
1 \\
2 \\
12 \\
4 \\
4 \\
4 \\
5 \\
2\end{array}$ & $\begin{array}{l}- \\
\overline{-} \\
\overline{-} \\
\overline{3} \\
- \\
\overline{-} \\
\overline{1} \\
-\end{array}$ & $\begin{array}{l}- \\
+ \\
\overline{-} \\
\overline{1} \\
1 \\
+ \\
+ \\
+ \\
+ \\
+ \\
-\end{array}$ & $\begin{array}{l}- \\
+ \\
- \\
\overline{-} \\
- \\
- \\
+ \\
+ \\
1 \\
-\end{array}$ & $\begin{array}{l}1 \\
1 \\
+ \\
1 \\
1 \\
3 \\
+ \\
5 \\
3 \\
+ \\
-\end{array}$ & $\begin{array}{r}4 \\
12 \\
5 \\
7 \\
11 \\
- \\
- \\
- \\
- \\
- \\
-\end{array}$ & $\begin{array}{l}6 \\
6 \\
2 \\
3 \\
7 \\
5 \\
2 \\
3 \\
1 \\
1 \\
3\end{array}$ & $\begin{array}{l}\overline{-} \\
\overline{-} \\
\overline{-} \\
\overline{-} \\
\overline{-} \\
\overline{-} \\
\overline{-}\end{array}$ & $\begin{array}{l}\overline{1} \\
1 \\
\overline{2} \\
2 \\
+ \\
1 \\
1 \\
1 \\
1\end{array}$ & $\begin{array}{l}- \\
- \\
- \\
- \\
- \\
- \\
\bar{\oplus} \\
4 \\
18 \\
16\end{array}$ \\
\hline & $\mathrm{a}$ & $\begin{array}{l}\text { Hole } 572 \mathrm{D} \\
\qquad \begin{array}{l}1, \mathrm{CC} \\
2-2,70-72 \\
2, \mathrm{CC} \\
3, \mathrm{CC}\end{array}\end{array}$ & $\begin{array}{l}156.3 \\
162.7 \\
170.3 \\
179.7 \\
\end{array}$ & $\begin{array}{l}\overline{-} \\
\overline{-}\end{array}$ & $\begin{array}{l}\overline{1} \\
+ \\
1\end{array}$ & $\begin{array}{l}1 \\
- \\
-\end{array}$ & $\begin{array}{l}1 \\
1 \\
+ \\
1\end{array}$ & $\begin{array}{l}\bar{z} \\
\overline{-}\end{array}$ & $\begin{array}{l}\overline{1} \\
1 \\
-\end{array}$ & $\begin{array}{l}1 \\
1 \\
-1 \\
1\end{array}$ & $\begin{array}{l}+ \\
1 \\
+ \\
1\end{array}$ & $\begin{array}{l}1 \\
1 \\
+ \\
+\end{array}$ & $\begin{array}{l}3 \\
1 \\
1 \\
4\end{array}$ & $\begin{array}{l}\overline{-} \\
\overline{-}\end{array}$ & $\begin{array}{l}1 \\
+ \\
- \\
-\end{array}$ & $\begin{array}{l}\overline{-} \\
\overline{-}\end{array}$ & $\begin{array}{l}+ \\
1 \\
1 \\
2\end{array}$ & $\begin{array}{l}\bar{z} \\
\overline{-}\end{array}$ & $\begin{array}{r}1 \\
2 \\
+ \\
1\end{array}$ & $\begin{array}{l}- \\
- \\
-\end{array}$ & $\begin{array}{l}1 \\
1 \\
+ \\
1\end{array}$ & $\begin{array}{r}15 \\
12 \\
11 \\
7\end{array}$ \\
\hline $\begin{array}{l}\text { Nitzschia } \\
\text { miocenica }\end{array}$ & b & $4, \mathrm{CC}$ & 189.0 & + & - & - & 2 & - & - & 2 & 1 & - & 1 & - & - & - & + & - & 2 & - & 2 & 9 \\
\hline
\end{tabular}

Notes: The abundance of each species is rounded to the nearest percentage. Samples are correlated to the diatom zonation of Burckle (1972, 1977). The age of each sample is extrapolated from the sediment accumulation curve. $+=\langle 1 \%$ of the assemblage; $\oplus=$ reworked specimens composing $<1 \%$ of the assemblage. 
Table 1. (Continued).

\begin{tabular}{|c|c|c|c|c|c|c|c|c|c|c|c|c|c|c|c|c|c|c|c|c|c|c|c|}
\hline $\begin{array}{l}\vdots \\
\frac{1}{2} \\
\frac{5}{2} \\
z\end{array}$ & 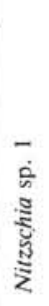 & 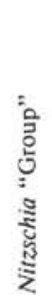 & 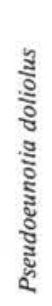 & 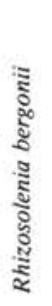 & 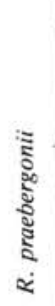 & 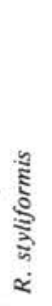 & 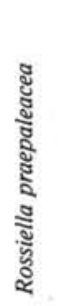 & 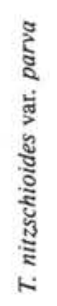 & 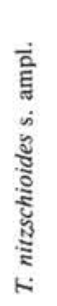 & 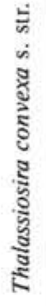 & 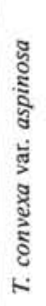 & 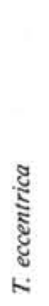 & $\begin{array}{l}\frac{y}{2} \\
\frac{0}{2} \\
\frac{0}{2} \\
\end{array}$ & 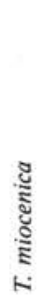 & 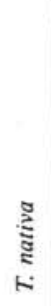 & 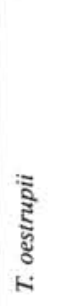 & 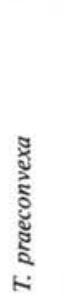 & 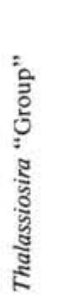 & 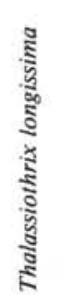 & 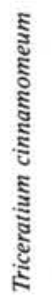 & 壱 & $\begin{array}{c}\text { Total } \\
\text { number } \\
\text { counted }\end{array}$ & $\begin{array}{c}\text { Extrapolated } \\
\text { age } \\
\text { (Ma) }\end{array}$ \\
\hline $\begin{array}{l}3 \\
1 \\
1 \\
3 \\
3 \\
\end{array}$ & $\begin{array}{l}- \\
\bar{y} \\
\overline{-} \\
-\end{array}$ & $\begin{array}{l}3 \\
2 \\
2 \\
4 \\
3\end{array}$ & $\begin{array}{l}8 \\
2 \\
6 \\
7 \\
3\end{array}$ & $\begin{array}{l}3 \\
3 \\
2 \\
1 \\
+\end{array}$ & $\begin{array}{l}4 \\
4 \\
4 \\
2 \\
1\end{array}$ & $\begin{array}{l}3 \\
2 \\
3 \\
1 \\
1 \\
\end{array}$ & $\begin{array}{l}- \\
\overline{-} \\
\overline{-}\end{array}$ & $\begin{array}{r}2 \\
9 \\
5 \\
2 \\
13\end{array}$ & $\begin{array}{r}15 \\
5 \\
15 \\
12 \\
13 \\
\end{array}$ & $\begin{array}{l}- \\
\overline{-} \\
- \\
-\end{array}$ & $\begin{array}{l}- \\
\overline{-} \\
\overline{-} \\
-\end{array}$ & $\begin{array}{l}3 \\
5 \\
7 \\
4 \\
4 \\
\end{array}$ & $\begin{array}{l}2 \\
3 \\
2 \\
5 \\
2 \\
\end{array}$ & $\begin{array}{l}- \\
\overline{-} \\
\overline{-}\end{array}$ & $\begin{array}{l}- \\
- \\
- \\
-\end{array}$ & $\begin{array}{l}19 \\
16 \\
16 \\
13 \\
13 \\
\end{array}$ & $\begin{array}{l}- \\
\overline{-} \\
\overline{-}\end{array}$ & $\begin{array}{l}1 \\
2 \\
2 \\
1 \\
1 \\
\end{array}$ & $\begin{array}{r}7 \\
12 \\
10 \\
4 \\
6 \\
\end{array}$ & $\begin{array}{l}4 \\
1 \\
2 \\
4 \\
2 \\
\end{array}$ & $\begin{array}{l}- \\
2 \\
1 \\
1 \\
1 \\
\end{array}$ & $\begin{array}{l}309 \\
293 \\
296 \\
299 \\
301 \\
\end{array}$ & $\begin{array}{l} \\
1.50 \\
1.62 \\
1.66 \\
1.70 \\
1.78 \\
\end{array}$ \\
\hline $\begin{array}{l}+ \\
+ \\
1 \\
3 \\
1 \\
2 \\
2 \\
3 \\
4 \\
+ \\
2 \\
+\end{array}$ & $\begin{array}{l}1 \\
1 \\
- \\
- \\
- \\
- \\
- \\
- \\
- \\
-\end{array}$ & $\begin{array}{r}3 \\
2 \\
3 \\
12 \\
6 \\
5 \\
8 \\
4 \\
2 \\
3 \\
1 \\
3\end{array}$ & $\begin{array}{l}- \\
- \\
- \\
- \\
- \\
- \\
- \\
z \\
- \\
-\end{array}$ & $\begin{array}{l}2 \\
2 \\
+ \\
1 \\
2 \\
+ \\
+ \\
+ \\
+ \\
+ \\
1 \\
1\end{array}$ & $\begin{array}{l}4 \\
2 \\
4 \\
4 \\
7 \\
3 \\
2 \\
3 \\
4 \\
3 \\
4 \\
5\end{array}$ & $\begin{array}{l}- \\
3 \\
+ \\
+ \\
1 \\
- \\
- \\
- \\
- \\
- \\
+ \\
1\end{array}$ & $\begin{array}{l}\overline{-} \\
\overline{-} \\
\overline{-} \\
\overline{-} \\
\overline{-} \\
\overline{-} \\
\overline{-} \\
-\end{array}$ & $\begin{array}{r}7 \\
5 \\
11 \\
5 \\
5 \\
14 \\
17 \\
9 \\
2 \\
23 \\
20 \\
12\end{array}$ & $\begin{array}{l}21 \\
20 \\
17 \\
18 \\
15 \\
26 \\
22 \\
21 \\
15 \\
17 \\
15 \\
23\end{array}$ & $\begin{array}{l}- \\
- \\
4 \\
\overline{2} \\
1 \\
1 \\
1 \\
1 \\
+ \\
3 \\
4 \\
4\end{array}$ & $\begin{array}{c}- \\
+ \\
3 \\
+ \\
6 \\
4 \\
7 \\
2 \\
1 \\
3 \\
8 \\
6\end{array}$ & $\begin{array}{l}6 \\
3 \\
4 \\
2 \\
3 \\
4 \\
6 \\
6 \\
4 \\
2 \\
2 \\
3\end{array}$ & $\begin{array}{l}4 \\
2 \\
1 \\
3 \\
3 \\
2 \\
3 \\
2 \\
2 \\
1 \\
1 \\
1\end{array}$ & $\begin{array}{l}- \\
\overline{-} \\
\overline{-} \\
\overline{-} \\
\overline{-} \\
- \\
\overline{-} \\
- \\
-\end{array}$ & $\begin{array}{l}- \\
\overline{-} \\
\overline{-} \\
- \\
- \\
- \\
- \\
- \\
- \\
-\end{array}$ & $\begin{array}{c}4 \\
3 \\
5 \\
5 \\
3 \\
4 \\
+ \\
5 \\
3 \\
1 \\
2 \\
3\end{array}$ & $\begin{array}{l}- \\
\overline{-} \\
\overline{-} \\
\overline{-} \\
\overline{-} \\
\overline{-} \\
\overline{-} \\
\overline{-}\end{array}$ & $\begin{array}{l}2 \\
1 \\
3 \\
2 \\
1 \\
2 \\
1 \\
+ \\
1 \\
1 \\
+ \\
-\end{array}$ & $\begin{array}{r}8 \\
11 \\
16 \\
5 \\
6 \\
2 \\
3 \\
5 \\
9 \\
12 \\
8 \\
12\end{array}$ & $\begin{array}{l}1 \\
2 \\
- \\
2 \\
4 \\
1 \\
2 \\
1 \\
- \\
- \\
+ \\
-\end{array}$ & $\begin{array}{l}1 \\
1 \\
2 \\
1 \\
1 \\
1 \\
+ \\
\overline{1} \\
1 \\
1 \\
+\end{array}$ & $\begin{array}{l}304 \\
302 \\
303 \\
294 \\
311 \\
299 \\
306 \\
298 \\
309 \\
294 \\
304 \\
284\end{array}$ & $\begin{array}{l}1.90 \\
2.23 \\
2.26 \\
2.33 \\
2.43 \\
2.50 \\
2.60 \\
2.70 \\
2.80 \\
2.87 \\
2.92 \\
3.00\end{array}$ \\
\hline $\begin{array}{l}8 \\
1 \\
3 \\
1 \\
- \\
+ \\
2 \\
2 \\
3 \\
2 \\
1 \\
- \\
1 \\
1 \\
- \\
+ \\
1 \\
+\end{array}$ & $\begin{array}{l}- \\
3 \\
+ \\
3 \\
- \\
2 \\
1 \\
2 \\
4 \\
3 \\
4 \\
- \\
- \\
- \\
+ \\
- \\
- \\
-\end{array}$ & $\begin{array}{l}1 \\
2 \\
3 \\
1 \\
1 \\
1 \\
1 \\
+ \\
1 \\
1 \\
1 \\
4 \\
1 \\
3 \\
1 \\
1 \\
2\end{array}$ & $\begin{array}{l}- \\
- \\
- \\
- \\
- \\
- \\
- \\
- \\
- \\
- \\
- \\
- \\
- \\
- \\
- \\
- \\
-\end{array}$ & $\begin{array}{l}6 \\
1 \\
1 \\
+ \\
- \\
1 \\
1 \\
1 \\
1 \\
+ \\
+ \\
2 \\
1 \\
- \\
- \\
+ \\
- \\
-\end{array}$ & $\begin{array}{l}\bar{z} \\
\bar{z} \\
\bar{z} \\
\overline{-} \\
\overline{-} \\
\overline{-} \\
\overline{-} \\
\overline{-} \\
\overline{-} \\
-\end{array}$ & $\begin{array}{l}1 \\
1 \\
- \\
- \\
- \\
- \\
- \\
- \\
+ \\
+ \\
+ \\
1 \\
- \\
+ \\
+ \\
1 \\
-\end{array}$ & $\begin{array}{l}\overline{-} \\
\overline{-} \\
\overline{-} \\
\overline{-} \\
\overline{-} \\
\overline{-} \\
\overline{-} \\
\overline{-} \\
\overline{-} \\
\overline{-} \\
\overline{-}\end{array}$ & $\begin{array}{r}6 \\
11 \\
8 \\
7 \\
16 \\
6 \\
5 \\
8 \\
14 \\
9 \\
13 \\
29 \\
12 \\
24 \\
20 \\
37 \\
26 \\
70\end{array}$ & $\begin{array}{r}7 \\
34 \\
9 \\
25 \\
31 \\
39 \\
22 \\
36 \\
16 \\
18 \\
14 \\
15 \\
35 \\
16 \\
37 \\
15 \\
33 \\
6\end{array}$ & $\begin{array}{l}-1 \\
1 \\
9 \\
8 \\
1 \\
3 \\
+ \\
- \\
- \\
- \\
- \\
- \\
- \\
- \\
- \\
- \\
- \\
-\end{array}$ & $\begin{array}{c}1 \\
+ \\
20 \\
11 \\
6 \\
8 \\
1 \\
3 \\
1 \\
1 \\
- \\
1 \\
- \\
+ \\
-1 \\
1 \\
+ \\
+\end{array}$ & $\begin{array}{l}3 \\
5 \\
3 \\
3 \\
1 \\
1 \\
4 \\
4 \\
5 \\
8 \\
4 \\
4 \\
1 \\
4 \\
5 \\
3 \\
4 \\
1\end{array}$ & $\begin{array}{l}2 \\
3 \\
1 \\
2 \\
+ \\
1 \\
1 \\
2 \\
3 \\
1 \\
1 \\
1 \\
+ \\
1 \\
1 \\
+ \\
1 \\
-\end{array}$ & $\begin{array}{l}\overline{-} \\
\overline{-} \\
\overline{-} \\
\overline{-} \\
\overline{-} \\
\overline{-} \\
- \\
- \\
\overline{-} \\
\overline{-} \\
\overline{-} \\
-\end{array}$ & $\begin{array}{l}- \\
- \\
- \\
- \\
- \\
- \\
- \\
- \\
+ \\
- \\
- \\
- \\
- \\
- \\
- \\
-\end{array}$ & $\begin{array}{l}4 \\
4 \\
- \\
3 \\
5 \\
2 \\
2 \\
3 \\
2 \\
4 \\
1 \\
2 \\
1 \\
3 \\
1 \\
3 \\
2 \\
2\end{array}$ & $\begin{array}{l}- \\
\overline{-} \\
\overline{-} \\
- \\
\overline{-} \\
\overline{-} \\
\overline{-} \\
\overline{-} \\
\overline{-} \\
\overline{-} \\
\overline{-} \\
-\end{array}$ & $\begin{array}{l}1 \\
1 \\
2 \\
1 \\
- \\
+ \\
3 \\
1 \\
4 \\
3 \\
3 \\
2 \\
+ \\
1 \\
2 \\
1 \\
1 \\
-\end{array}$ & $\begin{array}{r}6 \\
6 \\
12 \\
2 \\
10 \\
9 \\
1 \\
4 \\
4 \\
2 \\
4 \\
7 \\
13 \\
20 \\
14 \\
13 \\
6 \\
6\end{array}$ & $\begin{array}{l}1 \\
1 \\
2 \\
2 \\
- \\
+ \\
1 \\
- \\
+ \\
2 \\
1 \\
+ \\
1 \\
1 \\
2 \\
1 \\
+ \\
-\end{array}$ & $\begin{array}{l}2 \\
1 \\
+ \\
1 \\
1 \\
- \\
1 \\
1 \\
+ \\
1 \\
1 \\
2 \\
+ \\
2 \\
1 \\
1 \\
1 \\
+\end{array}$ & $\begin{array}{l}301 \\
298 \\
303 \\
294 \\
253 \\
275 \\
294 \\
305 \\
311 \\
306 \\
298 \\
301 \\
296 \\
293 \\
300 \\
306 \\
304 \\
271\end{array}$ & $\begin{array}{l}3.10 \\
3.20 \\
3.27 \\
3.37 \\
3.43 \\
3.47 \\
3.56 \\
3.61 \\
3.63 \\
3.70 \\
3.73 \\
3.74 \\
3.83 \\
3.94 \\
4.04 \\
4.18 \\
4.27 \\
4.41\end{array}$ \\
\hline $\begin{array}{l}1 \\
1 \\
- \\
1 \\
1 \\
1 \\
1 \\
2 \\
1 \\
2 \\
1\end{array}$ & $\begin{array}{l}- \\
\bar{z} \\
\overline{-} \\
\overline{-} \\
\bar{z} \\
\overline{-} \\
-\end{array}$ & $\begin{array}{c}1 \\
2 \\
+ \\
2 \\
2 \\
1 \\
1 \\
1 \\
2 \\
5 \\
1\end{array}$ & $\begin{array}{l}- \\
- \\
- \\
- \\
- \\
- \\
- \\
- \\
- \\
- \\
-\end{array}$ & $\begin{array}{l}- \\
- \\
- \\
- \\
- \\
+ \\
1 \\
\overline{1} \\
+ \\
-\end{array}$ & $\begin{array}{l}\overline{-} \\
\overline{-} \\
\overline{-} \\
\overline{-} \\
\overline{-} \\
\overline{-} \\
\overline{-}\end{array}$ & $\begin{array}{l}+ \\
- \\
1 \\
- \\
- \\
2 \\
- \\
- \\
- \\
- \\
-\end{array}$ & $\begin{array}{l}\overline{-} \\
\overline{-} \\
\overline{-} \\
\overline{-} \\
\overline{-} \\
\overline{-} \\
\overline{-} \\
-\end{array}$ & $\begin{array}{r}20 \\
27 \\
50 \\
8 \\
11 \\
18 \\
14 \\
31 \\
8 \\
30 \\
31\end{array}$ & $\begin{array}{l}38 \\
26 \\
27 \\
49 \\
38 \\
32 \\
53 \\
24 \\
41 \\
14 \\
22\end{array}$ & $\begin{array}{l}- \\
\overline{-} \\
\overline{-} \\
\overline{-} \\
\overline{-} \\
- \\
-\end{array}$ & $\begin{array}{l}+ \\
- \\
+ \\
1 \\
- \\
+ \\
1 \\
3 \\
3 \\
3 \\
3\end{array}$ & $\begin{array}{l}4 \\
2 \\
2 \\
2 \\
3 \\
6 \\
3 \\
5 \\
5 \\
3 \\
2\end{array}$ & $\begin{array}{l}1 \\
1 \\
+ \\
+ \\
3 \\
1 \\
- \\
+ \\
+ \\
1 \\
1\end{array}$ & $\begin{array}{l}- \\
- \\
- \\
- \\
- \\
+ \\
3 \\
2 \\
7 \\
1 \\
2\end{array}$ & $\begin{array}{l}+ \\
- \\
\overline{1} \\
- \\
\overline{2} \\
\overline{2} \\
+ \\
+\end{array}$ & \begin{tabular}{|c|}
3 \\
5 \\
4 \\
5 \\
5 \\
- \\
- \\
-- \\
- \\
- \\
-
\end{tabular} & $\begin{array}{l}- \\
- \\
- \\
- \\
- \\
- \\
- \\
- \\
- \\
- \\
-\end{array}$ & $\begin{array}{l}\overline{1} \\
\overline{1} \\
1 \\
1 \\
+ \\
1 \\
1 \\
1 \\
1 \\
1\end{array}$ & $\begin{array}{r}11 \\
4 \\
3 \\
16 \\
5 \\
5 \\
4 \\
7 \\
6 \\
9 \\
4 \\
7\end{array}$ & $\begin{array}{l}- \\
1 \\
+ \\
1 \\
1 \\
3 \\
1 \\
1 \\
- \\
- \\
1\end{array}$ & $\begin{array}{l}1 \\
3 \\
2 \\
1 \\
1 \\
1 \\
2 \\
2 \\
2 \\
2\end{array}$ & $\begin{array}{l}295 \\
298 \\
297 \\
296 \\
295 \\
303 \\
303 \\
299 \\
306 \\
306 \\
293\end{array}$ & $\begin{array}{l}4.50 \\
4.64 \\
4.87 \\
4.95 \\
5.08 \\
5.30 \\
5.40 \\
5.53 \\
5.58 \\
5.64 \\
5.76\end{array}$ \\
\hline $\begin{array}{l}+ \\
1 \\
+ \\
1\end{array}$ & $\begin{array}{l}\overline{-} \\
\overline{-}\end{array}$ & $\begin{array}{c}1 \\
\frac{1}{2} \\
+\end{array}$ & $\begin{array}{l}\bar{z} \\
\overline{-}\end{array}$ & $\begin{array}{l}\overline{-} \\
\overline{+} \\
+\end{array}$ & $\begin{array}{l}- \\
- \\
-\end{array}$ & $\begin{array}{l}\overline{2} \\
- \\
-\end{array}$ & $\begin{array}{l}- \\
+ \\
\overline{1}\end{array}$ & $\begin{array}{l}41 \\
31 \\
37 \\
21\end{array}$ & $\begin{array}{r}9 \\
22 \\
24 \\
22\end{array}$ & $\begin{array}{l}- \\
\overline{-} \\
-\end{array}$ & $\begin{array}{l}3 \\
4 \\
1 \\
1\end{array}$ & $\begin{array}{l}2 \\
2 \\
2 \\
1\end{array}$ & $\begin{array}{l}- \\
1 \\
1\end{array}$ & $\begin{array}{l}2 \\
1 \\
1 \\
-\end{array}$ & $\begin{array}{l}+ \\
\pm \\
-\end{array}$ & $\begin{array}{l}- \\
- \\
-\end{array}$ & $\begin{array}{l}1 \\
2 \\
\overline{1}\end{array}$ & $\begin{array}{l}1 \\
\frac{1}{1}\end{array}$ & $\begin{array}{r}12 \\
9 \\
13 \\
27\end{array}$ & $\begin{array}{l}+ \\
+ \\
+ \\
+\end{array}$ & $\begin{array}{l}1 \\
2 \\
1 \\
3\end{array}$ & $\begin{array}{l}274 \\
300 \\
292 \\
292\end{array}$ & $\begin{array}{l}5.79 \\
5.88 \\
5.98 \\
6.09\end{array}$ \\
\hline+ & - & - & - & - & - & + & 2 & 16 & 17 & - & - & 4 & + & - & + & - & 2 & 2 & 25 & - & 2 & 286 & 6.20 \\
\hline
\end{tabular}


Table 2. Relative frequency of species encountered during examination of samples from Hole 503A.

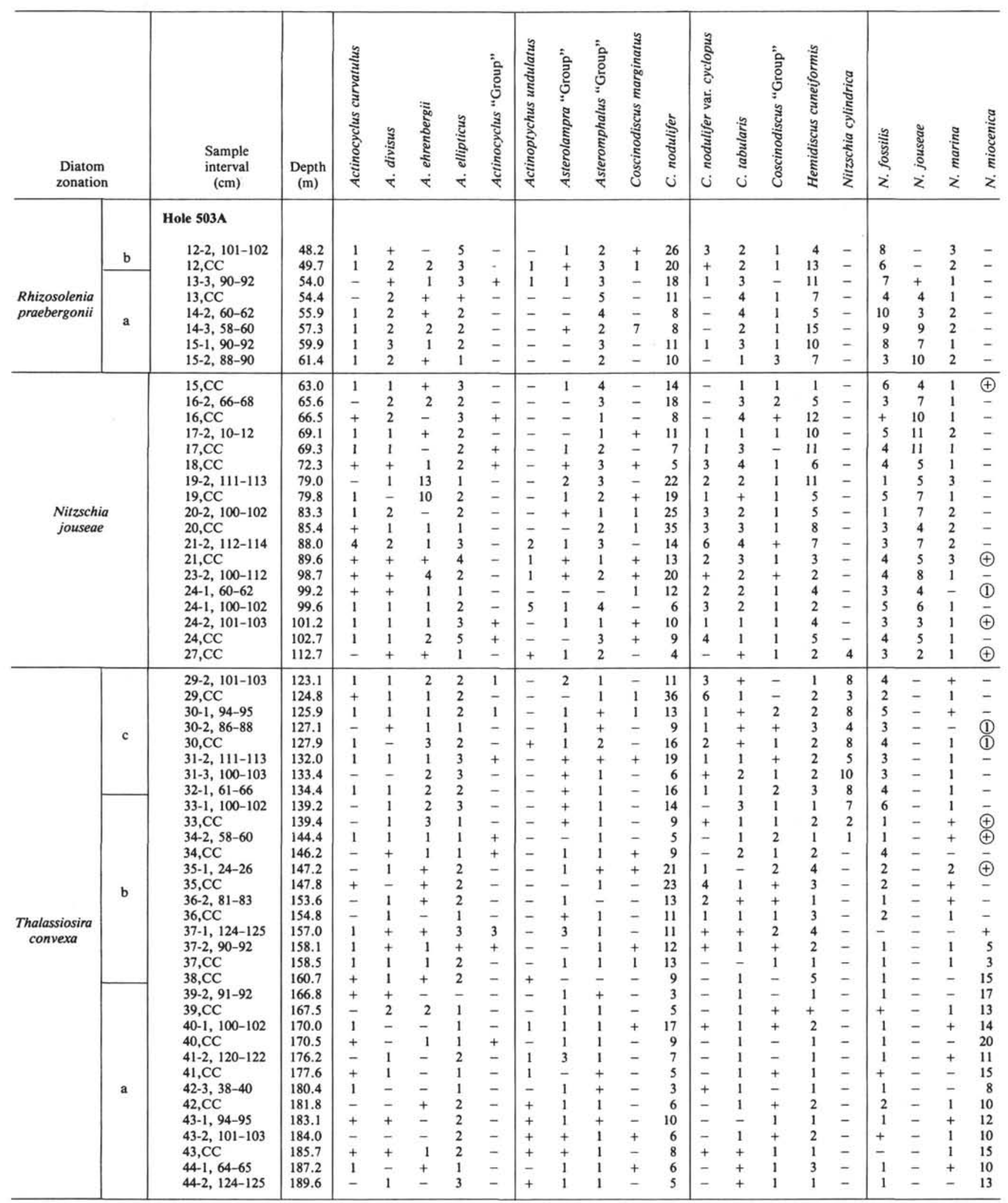

Notes: The depth of each sample is recorded in meters. The abundance of each species is rounded to the nearest percentage. Samples are correlated to the diatom zonation of Burckle $(1972,1977)$. The age of each sample is extrapolated from the sediment accumulation curve. $+=\langle 1 \%$ of the assemblage. $\oplus=$ reworked specimens composing $<1 \%$ of the assemblage; (1) $=$ reworked specimens composing $1 \%$ of the assemblage; (2) $=$ reworked specimens composing $2 \%$ of the assemblage. 
Table 2. (Continued).

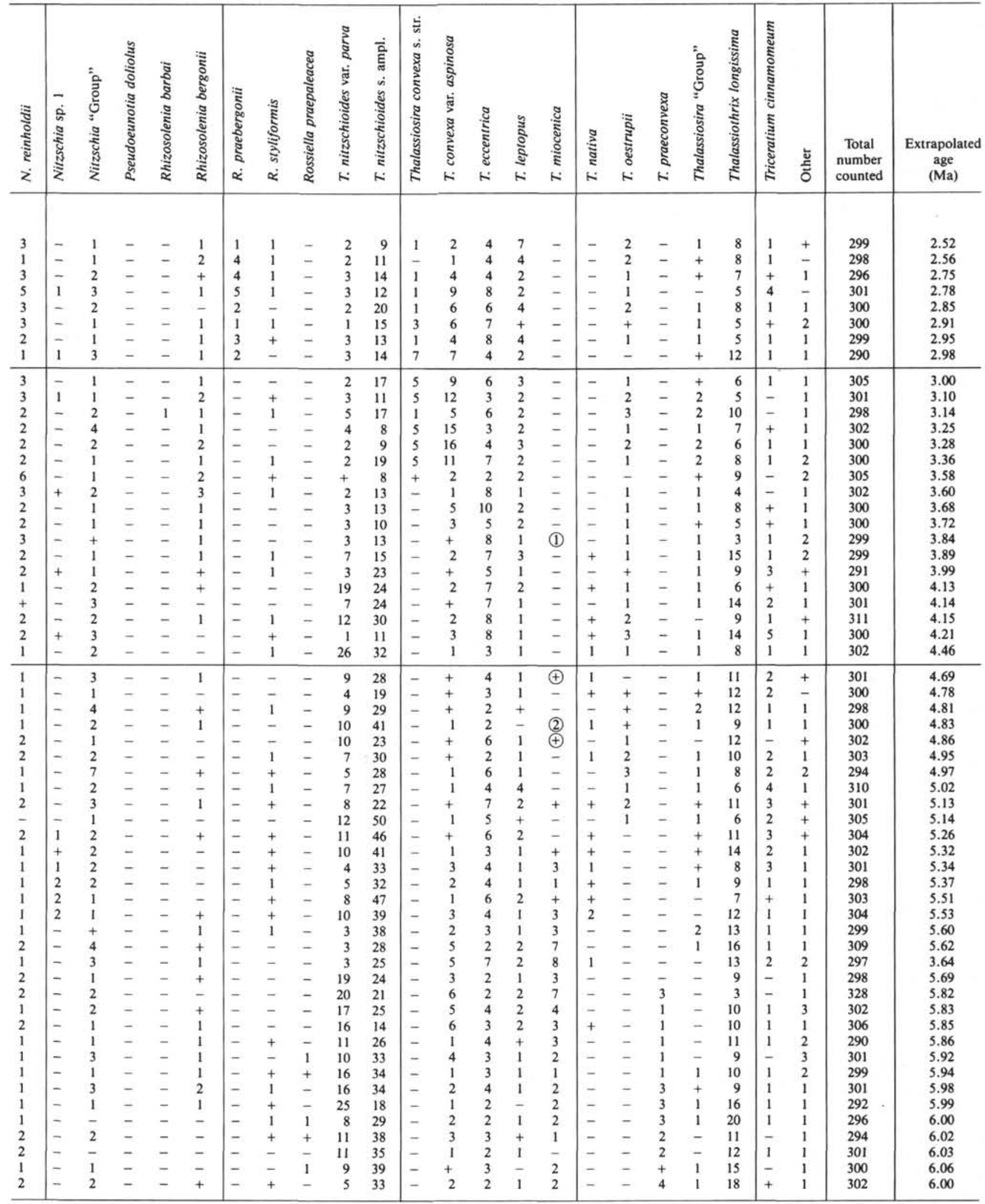


Table 3. Relative frequency of species encountered during examination of samples from Hole 573.

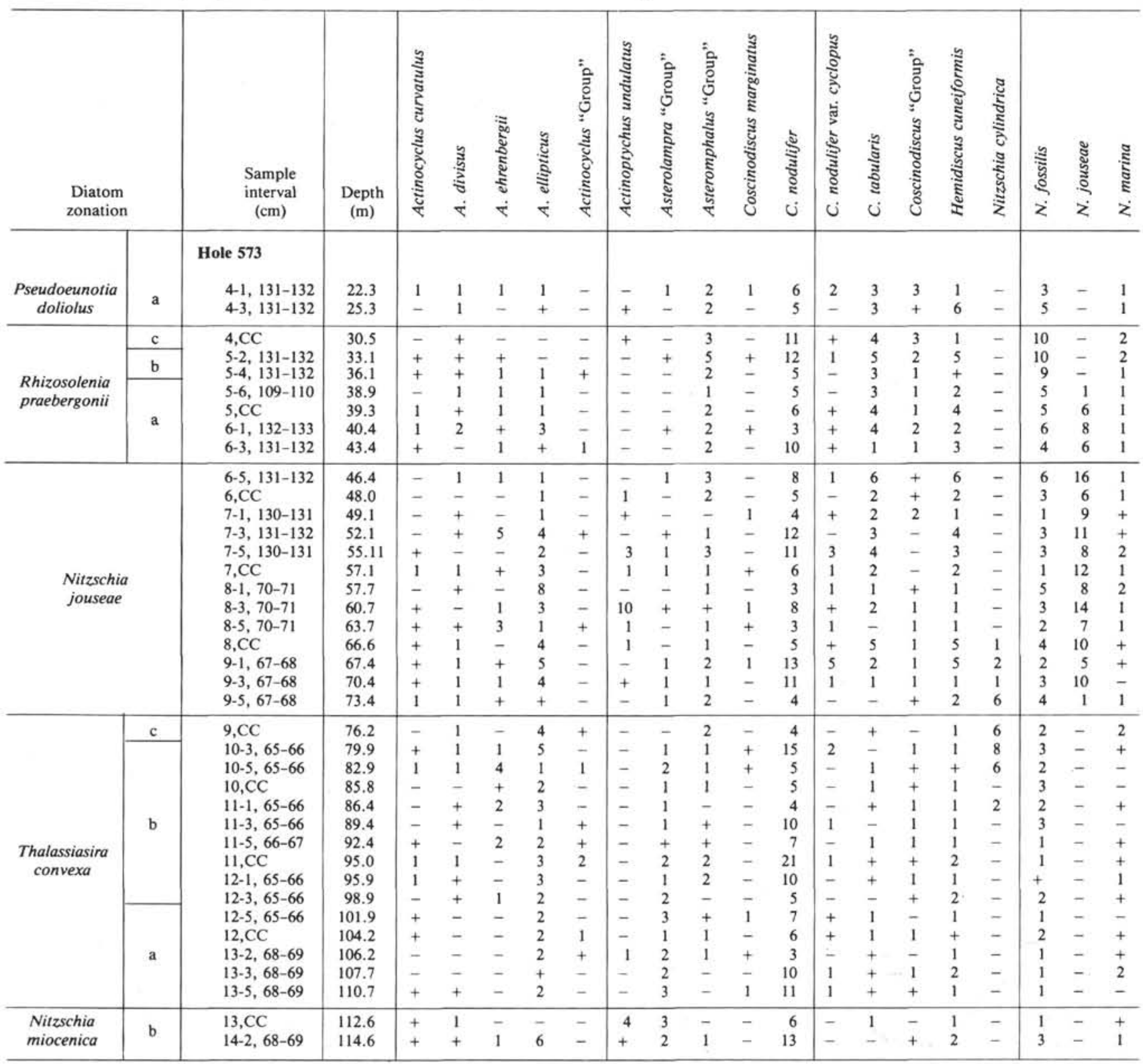

Notes: The abundance of each species is rounded to the nearest percentage. Samples are correlated to the diatom zonation of Burckle (1972, 1977). The depth of each sample is recorded in meters. The age of each sample is extrapolated from the sediment accumulation curve. $+=\langle 1 \%$ of the assemblage. $\oplus=$ reworked specimens composing $<1 \%$ of the assemblage.

age difference may result from paleoceanographic provincialism, which may be supported by examination of the abundance of Thalassiosira miocenica over the same time interval. At both Sites 572 and 503, T. miocenica increases approximately $5 \%$ between the peak in abundance and the last occurrence of $N$. miocenica. This slight increase in T. miocenica is also observed at Site 573 , but when compared to the range of $N$. miocenica at Sites 503 and 572, the increase occurs over a longer period of time and approximately $0.15 \mathrm{~m}$.y. prior to its occurrence at the other sites (Figs. 4 to 6). The peak in abundance of $N$. miocenica is therefore useful in the high productivity region of the equatorial Pacific and is assigned a tentative age of $5.6 \mathrm{Ma}$. The greater age (5.8 Ma) extrapolated for this event at Site 573 may be useful in the low productivity zone of the eastern equatorial Pacific. However, further documentation of this event is required before it can be considered stratigraphically useful in this region.

\section{Acme of Actinocyclus ehrenbergii}

Actinocyclus ehrenbergii typically composes $1 \%$ of the overall diatom assemblage (see Tables 1 to 3 ). However, in Sample 572A-7-2, 66-67 cm, 503A-19-2, 111$113 \mathrm{~cm}$, and 503A-19, CC, A. ehrenbergii attains 14,13 , and $10 \%$ of the overall assemblage, respectively. This acme of $A$. ehrenbergii approximates the first occurrence of Thalassiosira convexa var. convexa (Figs. 7 and 8). The extrapolated ages for these samples are $3.6 \mathrm{Ma}(572 \mathrm{~A}$ 7-2), 3.6 Ma (503A-19-2), and $3.60 \mathrm{Ma}(503 \mathrm{~A}-19, \mathrm{CC})$. 
Table 3. (Continued).

\begin{tabular}{|c|c|c|c|c|c|c|c|c|c|c|c|c|c|c|c|c|c|c|c|c|c|c|c|c|}
\hline 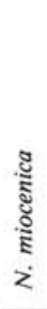 & 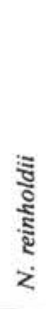 & 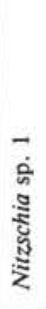 & 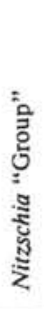 & 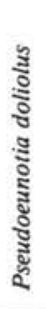 & 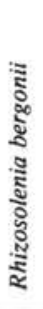 & 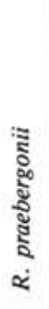 & 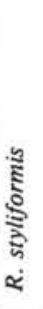 & 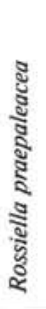 & 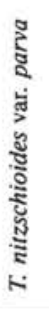 & 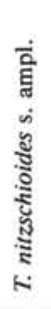 & 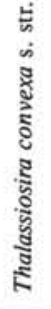 & 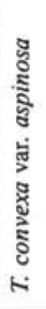 & 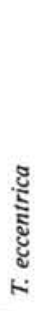 & 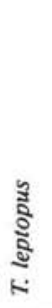 & 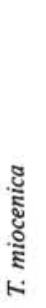 & 气ूँ & 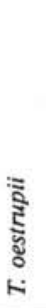 & 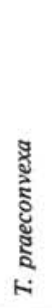 & 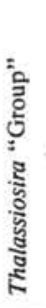 & 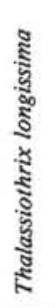 & 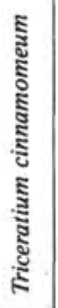 & さ્ & $\begin{array}{c}\text { Total } \\
\text { number } \\
\text { counted }\end{array}$ & $\begin{array}{c}\text { Extrapolated } \\
\text { age } \\
\text { (Ma) }\end{array}$ \\
\hline- & 3 & 4 & 3 & 1 & 3 & 1 & 1 & - & 8 & 11 & - & - & 8 & 1 & - & - & 13 & - & 1 & 6 & 3 & + & 300 & 1.50 \\
\hline- & 1 & 2 & 2 & 3 & 1 & 2 & 2 & - & 9 & 16 & - & - & 8 & 2 & - & - & 12 & - & 3 & 4 & 5 & 2 & 302 & 1.63 \\
\hline- & 3 & + & - & - & 2 & 14 & + & - & 3 & 15 & - & - & 12 & 2 & - & - & 1 & - & 1 & 10 & 3 & 1 & 302 & 2.10 \\
\hline- & 3 & 2 & 3 & - & 3 & 4 & - & - & 1 & 20 & - & + & 11 & 2 & - & - & 2 & - & 2 & 1 & 4 & 1 & 281 & 2.30 \\
\hline- & 1 & 13 & 1 & - & 1 & 3 & - & - & 7 & 28 & 1 & 2 & 5 & 3 & - & - & 3 & - & 3 & 3 & 2 & 1 & 303 & 2.50 \\
\hline- & 2 & 7 & 1 & - & 2 & 4 & + & - & - & 34 & 1 & 3 & 4 & 2 & - & - & 2 & - & 6 & 5 & 2 & 1 & 295 & 2.60 \\
\hline- & 3 & 3 & 1 & - & 2 & 5 & + & - & 11 & 19 & 1 & 4 & 7 & 2 & - & - & + & - & 2 & 6 & 2 & 1 & 297 & 2.73 \\
\hline- & 3 & 5 & 3 & - & 1 & 4 & - & - & 8 & 20 & 1 & 4 & 8 & 2 & - & - & 1 & - & 2 & 3 & 3 & + & 302 & 2.80 \\
\hline- & 2 & 2 & 1 & - & 3 & 1 & - & - & 7 & 13 & 8 & 10 & 5 & 2 & - & - & 2 & - & 1 & 7 & 1 & 1 & 292 & 2.97 \\
\hline- & 1 & 1 & 1 & - & 2 & - & - & - & - & 13 & 2 & 17 & 3 & 3 & - & - & 1 & - & + & 3 & 2 & + & 294 & 3.25 \\
\hline- & 1 & 3 & - & - & 1 & - & - & - & 10 & 41 & 3 & 7 & 3 & 1 & - & - & + & - & 1 & 5 & 2 & + & 296 & 3.38 \\
\hline- & + & 1 & 2 & - & 3 & - & + & - & 12 & 37 & 5 & 8 & 2 & 1 & - & - & 1 & - & + & 5 & 1 & 1 & 302 & 3.43 \\
\hline - & 1 & 1 & 1 & - & 2 & - & - & - & 8 & 24 & - & - & 3 & 1 & - & - & $i$ & - & 2 & 5 & 4 & 1 & 299 & 3.67 \\
\hline - & + & - & 3 & - & 2 & - & 7 & - & - & 25 & - & 3 & 4 & 3 & - & - & 1 & - & 2 & 5 & + & + & 300 & 3.90 \\
\hline - & 1 & 5 & 1 & - & 1 & - & + & - & 22 & 22 & - & 1 & 3 & 2 & - & - & 1 & - & 2 & 5 & 1 & + & 296 & 3.96 \\
\hline - & + & 7 & 3 & - & - & - & - & - & - & 35 & - & 1 & 5 & 1 & - & - & 2 & - & 2 & 6 & 2 & 3 & 295 & 4.00 \\
\hline - & + & - & 1 & - & + & - & 1 & - & 8 & 22 & - & 3 & 7 & 1 & - & - & 1 & - & + & 5 & 3 & 1 & 303 & 4.06 \\
\hline - & 1 & - & 1 & - & + & - & - & - & 19 & 38 & - & + & 2 & 1 & - & - & 2 & - & - & 10 & 4 & 1 & 302 & 4.15 \\
\hline - & - & 1 & + & - & - & - & 1 & - & 7 & 13 & - & 1 & 17 & 3 & - & - & 2 & - & 1 & 12 & 4 & 1 & 301 & 4.25 \\
\hline - & + & - & 1 & - & - & - & - & - & 2 & 16 & - & 6 & 7 & 2 & - & - & 3 & - & 3 & 8 & 5 & 2 & 300 & 4.28 \\
\hline - & $\overline{-}$ & 1 & 1 & - & - & - & - & - & 8 & 26 & - & 2 & 9 & 1 & - & - & 3 & - & 2 & 6 & 3 & + & 301 & 4.38 \\
\hline - & 2 & + & 1 & - & - & - & - & - & 11 & 26 & - & 1 & 7 & 1 & - & - & 2 & - & 3 & 9 & 1 & 1 & 303 & 4.47 \\
\hline - & 2 & - & - & - & - & - & - & - & 14 & 44 & - & 2 & 3 & + & - & - & + & - & 1 & 10 & 1 & - & 301 & 5.10 \\
\hline- & 1 & 2 & 1 & - & - & - & + & - & 2 & 36 & - & 1 & 5 & 2 & 1 & 1 & - & - & 1 & 5 & 1 & 1 & 299 & 5.20 \\
\hline$\oplus$ & 2 & 2 & 1 & - & - & - & 1 & - & 4 & 45 & - & 1 & 4 & 1 & 1 & 2 & - & - & 4 & 7 & 5 & 1 & 302 & 5.27 \\
\hline - & 1 & - & 6 & - & - & - & - & - & -4 & 65 & - & - & 2 & 1 & + & - & - & - & - & 6 & 1 & 1 & 301 & 5.35 \\
\hline$\oplus$ & 1 & - & 1 & - & - & - & - & - & - & 71 & - & - & 2 & 1 & - & 1 & - & - & 1 & 5 & 2 & - & 300 & 5.38 \\
\hline - & 2 & - & 2 & - & - & - & + & - & 12 & 47 & - & 1 & 6 & 3 & 2 & 1 & - & - & 1 & 5 & 1 & 1 & 300 & 5.44 \\
\hline - & 1 & 3 & 2 & - & - & - & - & - & 9 & 57 & - & 2 & 5 & - & 1 & - & - & - & 1 & 4 & 1 & + & 301 & 5.51 \\
\hline 2 & 1 & - & 1 & - & - & - & - & - & 4 & 22 & - & 1 & 7 & 3 & 3 & 1 & - & - & 2 & 13 & 2 & 1 & 302 & 5.60 \\
\hline 14 & 1 & - & 1 & - & - & - & 1 & - & 11 & 23 & - & 1 & 11 & 2 & 3 & - & - & - & 1 & 12 & 1 & - & 303 & 5.65 \\
\hline 16 & + & - & 2 & - & + & - & - & - & - & 36 & - & 4 & 3 & + & 8 & - & - & - & 3 & 7 & 3 & 1 & 287 & 5.75 \\
\hline 23 & 1 & 1 & - & - & - & - & - & - & 8 & 22 & - & 4 & 1 & + & 7 & + & - & 1 & 1 & 12 & - & 1 & 301 & 5.83 \\
\hline 16 & + & - & 1 & - & - & - & 1 & - & 17 & 20 & - & 2 & 5 & 1 & 5 & - & - & 4 & i & 8 & + & 2 & 302 & 5.88 \\
\hline 16 & 1 & - & 1 & - & + & - & - & + & 15 & 32 & - & 2 & 3 & + & 3 & - & - & 3 & + & 10 & 1 & 2 & 293 & 5.95 \\
\hline 12 & 1 & 2 & - & - & - & - & 1 & + & - & 32 & - & 2 & 3 & 2 & 3 & - & - & 3 & 1 & 19 & 1 & 1 & 302 & 5.98 \\
\hline 10 & 1 & - & 1 & - & 1 & - & 1 & + & 1 & 36 & - & 2 & 5 & 1 & - & + & - & 4 & 1 & 15 & 2 & 1 & 300 & 6.06 \\
\hline 15 & 1 & - & 1 & - & - & - & + & - & 5 & 36 & - & - & 5 & 1 & - & - & - & 3 & 1 & 11 & 2 & 1 & 299 & 6.12 \\
\hline 22 & 1 & - & 8 & - & - & - & + & + & 2 & 14 & - & - & 7 & 3 & - & - & - & - & 2 & 9 & 2 & 1 & 298 & 6.18 \\
\hline
\end{tabular}

Unfortunately, the geographic extent of this datum is presently unknown. This datum was not observed at Site 573 because no samples were examined from this time interval.

\section{First Peak in Abundance of Thalassiosira convexa Group}

Stratigraphically below the first occurrence of Thalassiosira convexa var. convexa at $3.6 \mathrm{Ma}, T$. convexa var. aspinosa commonly makes up less than $3 \%$ of the diatom assemblage. Directly above the first occurrence of $T$. convexa var. convexa, the abundance of $T$. convexa Group ( $T$. convexa var. aspinosa and $T$. convexa var. convexa) increases gradually from less than $3 \%$ at $3.6 \mathrm{Ma}$ to between 20 and $30 \%$ at $3.27 \mathrm{Ma}$. A sharp decline in abundance of $T$. convexa Group occurs in younger material. This peak in abundance is observed at all sites (Figs. 7 to 9) and has an extrapolated age of 3.25 $\mathrm{Ma}$ at Site 573 and of $3.27 \mathrm{Ma}$ at Sites 572 and 503 .

\section{Base of the Second Abundance Interval of Thalassiosira convexa Group}

This datum was originally defined by Burckle and Trainer (1979) as the "reoccurrent appearance" of Thalassiosira convexa. They placed this event directly below the first occurrence of Rhizosolenia praebergonii (3.0 $\mathrm{Ma}$ ) and within the Mammoth Subchron. An age of approximately $3.03 \mathrm{Ma}$ was assigned by Burckle and Trainer (1979) to this event. Adjustment of this date to more current ages given for the Mammoth Subchron (bottom at $3.17 \mathrm{Ma}$, top at $3.07 \mathrm{Ma}$; Berggren et al., in press) indicates that the age of this datum is most likely slightly older than originally estimated. 


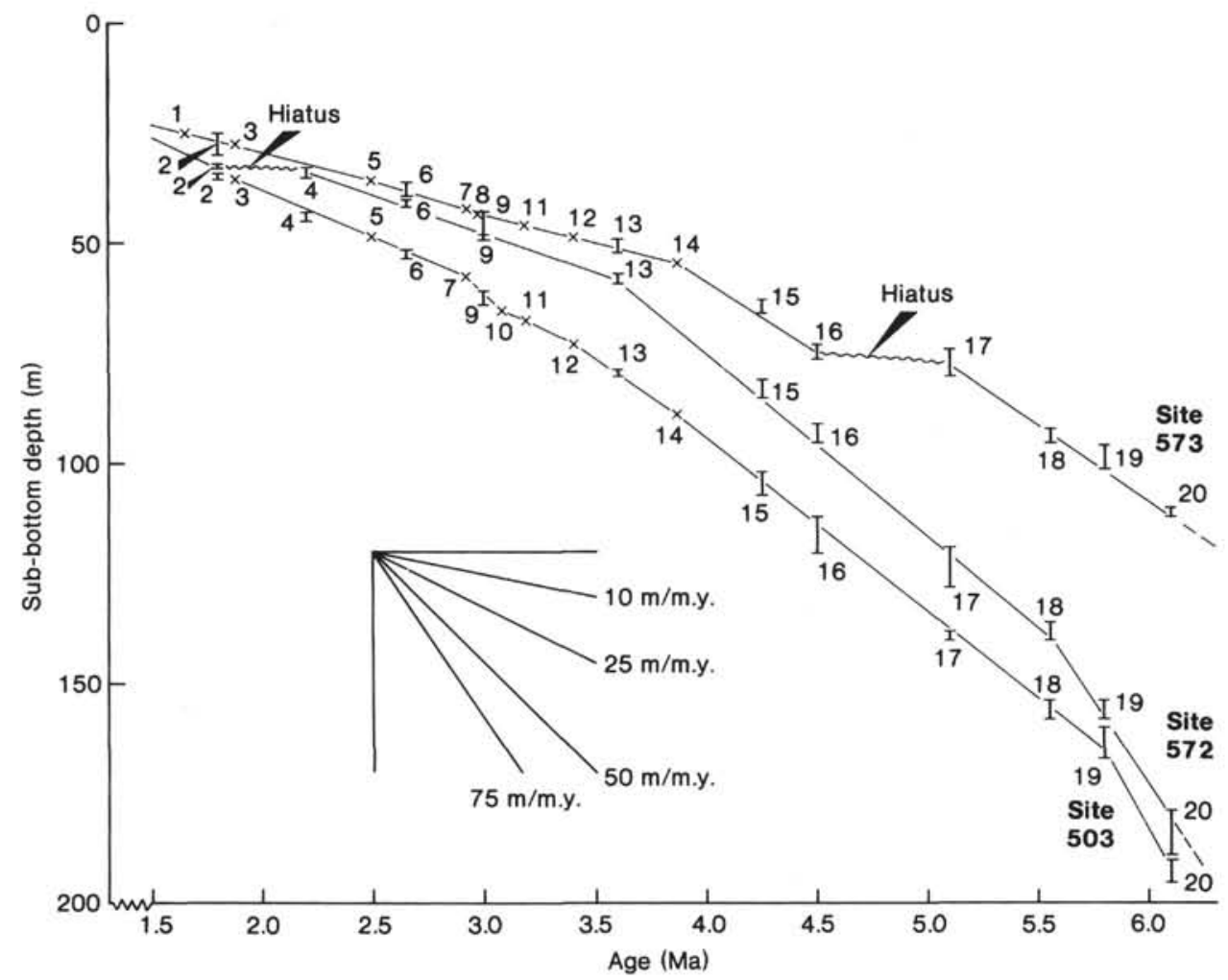

Figure 3. Sediment accumulation curves for Sites 572, 573, and 503. 1. top of Olduvai; 2. first $P$. doliolus; 3. base of Olduvai; 4 . last $T$. convexa; 5 . top of Gauss; 6 . last $N$. jouseae; 7. top of Kaena; 8 . base of Kaena; 9. first $R$. praebergonii; 10. top of Mammoth: 11. base of Mammoth; 12. Gilbert/Gauss boundary; 13. first $T$. convexa var. convexa; 14. top of Cochiti; 15 . last $N$. cyclindrica; 16 . first $N$. jouseae; 17. last $T$. miocenica; 18. last $N$. miocenica; 19. last $T$. praeconvexa; 20. first $T$. convexa and $T$. miocenica. $\times=$ magnetostratigraphic boundaries. Vertical bars indicate range of uncertainty in sample depth.

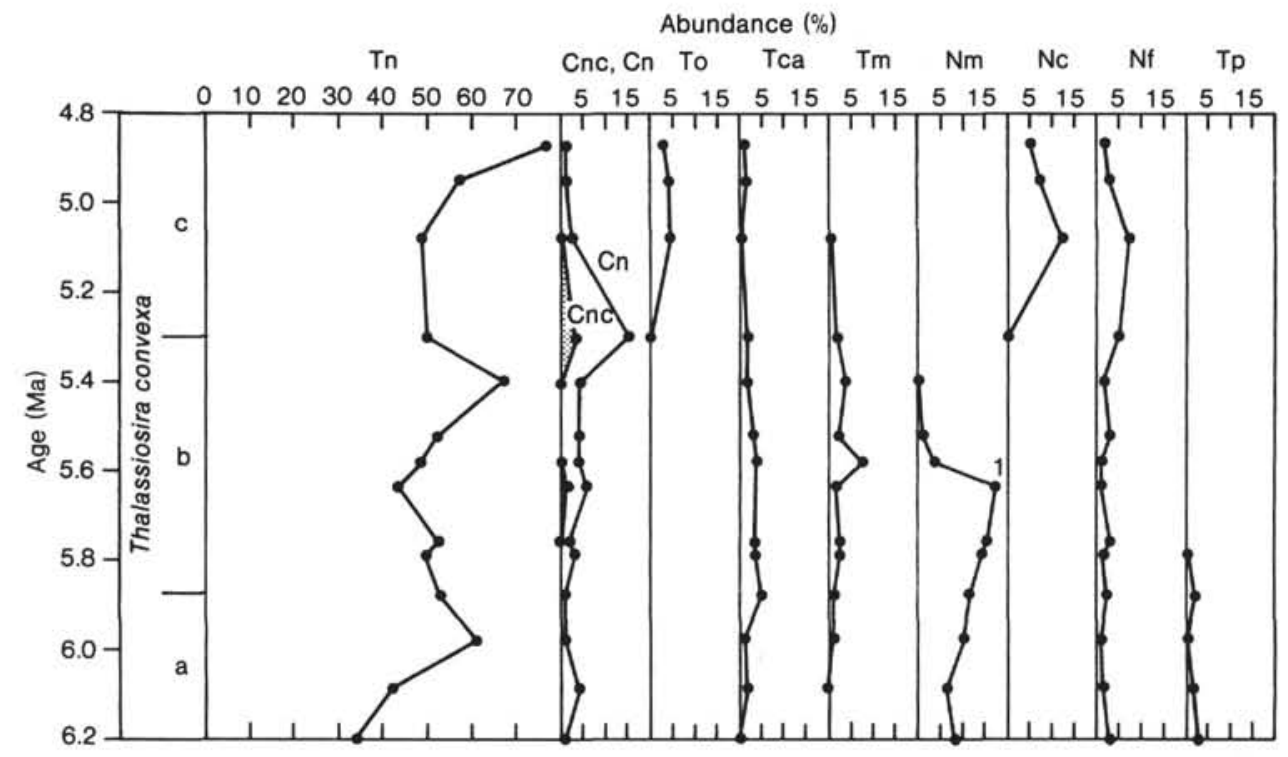

Figure 4. Fluctuations in abundance (percentage) of selected species from Site 572 for the interval 6.2 to $4.8 \mathrm{Ma} . \mathrm{Tn}=T$. nitzschioides $; \mathrm{Cnc}=C$. nodulifer var. cyclopus $; \mathrm{Cn}=C$. nodulifer; $\mathrm{To}=T$. oestrupii $; \mathrm{Tca}=T$. convexa var. aspinosa $; \mathrm{Tm}=T$. miocenica $; \mathrm{Nm}=N$. miocenica $(1$, peak in abundance of $N$. miocenica); $\mathrm{Nc}=N$. cylindrica s. str.; $\mathrm{Nf}=N$. fossilis; $\mathrm{Tp}=T$. praeconvexa. The diatom zones figured are from Burckle $(1972,1977)$. Ages of samples are extrapolated from the sediment accumulation curves (Fig. 3). 


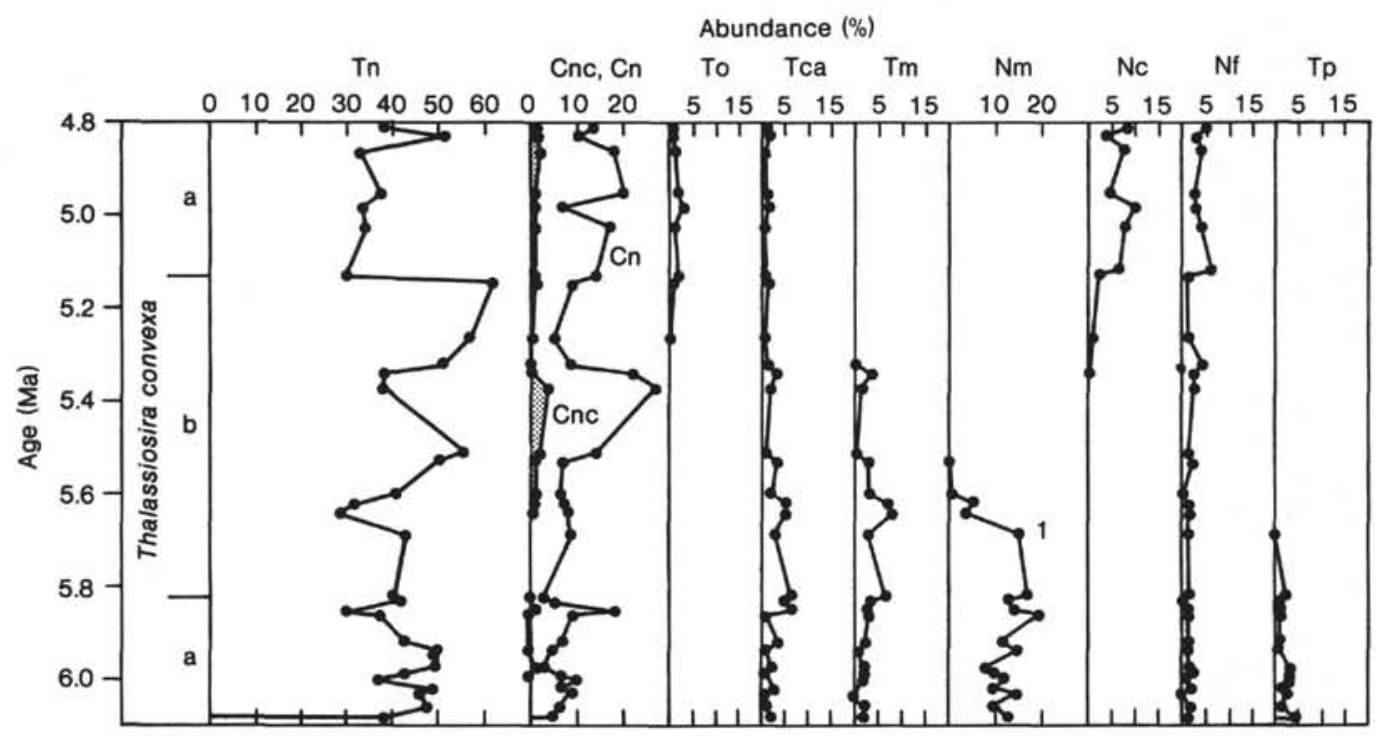

Figure 5. Fluctuations in abundance (percentage) of selected species from Site 503 for the interval 6.2 to $4.8 \mathrm{Ma}$. Abbreviations and notes same as for Figure 4.

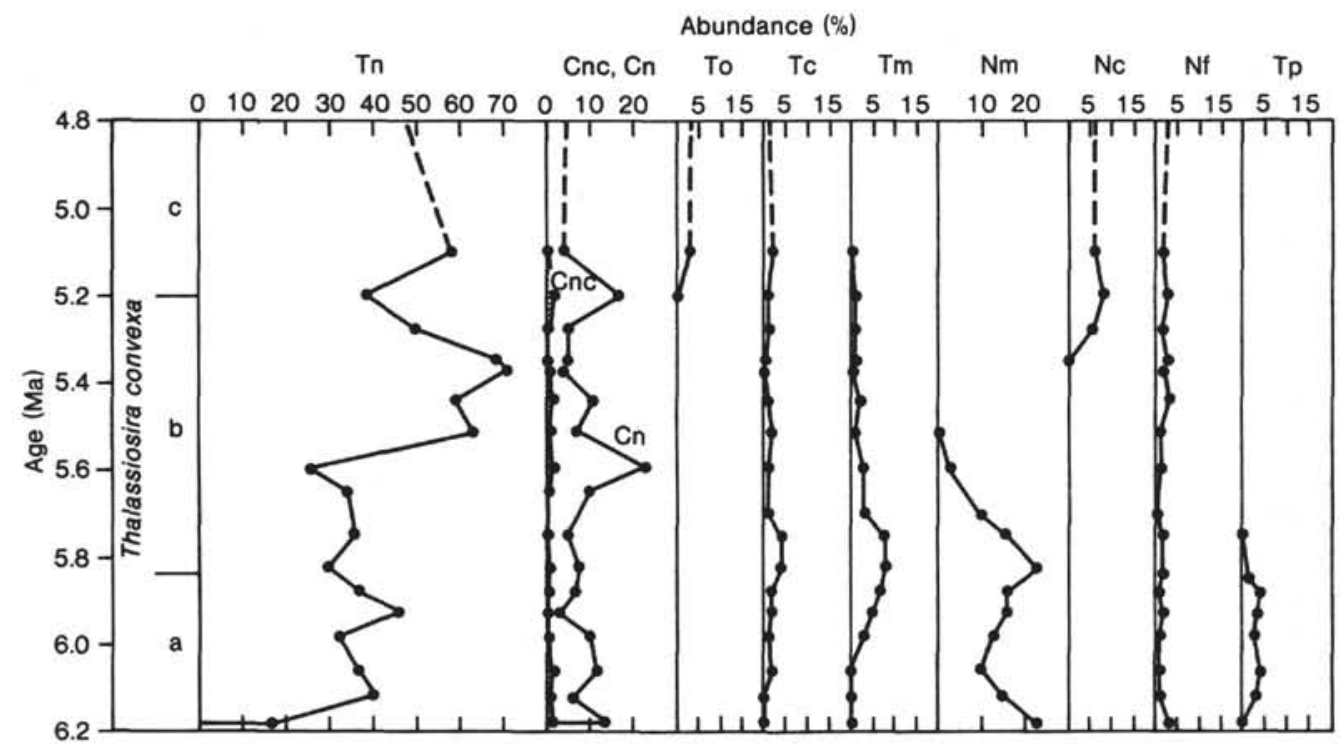

Figure 6. Fluctuations in abundance (percentage) of selected species from Site 573 for the interval 6.2 to 4.8 Ma. Dashed line represents duration of observed hiatus. Abbreviations and notes same as for Figure 4.

At Sites 572 (Fig. 10) and 503 (Fig. 11), a second abundance interval of $T$. convexa Group is observed between 2.9 and $3.1 \mathrm{Ma}$. The recurrence of $T$. convexa, as described by Burckle and Trainer (1979), corresponds to the base of this second abundance interval. At Site 572 the base of this second interval occurs between 3.0 and 3.1 Ma. At Site 503 this event occurs between 3.1 and $3.15 \mathrm{Ma}$. Therefore, a tentative age of $3.10 \mathrm{Ma}$ is assigned to this datum. As a result of the sampling interval used in this study, this event can not be recognized at Site 573.

Burckle and Trainer (1979, p. 255) noted that $T$. convexa disappears slightly above the Gilbert/Gauss bound- ary in Core V28-179 from the equatorial Pacific and suggested that this disappearance may be a useful secondary datum. They assigned an approximate age of $\mathbf{3 . 2}$ Ma to this event, indicating that it should occur between the first peak in abundance of $T$. convexa Group (3.27 $\mathrm{Ma}$ ) and the base of the second abundance interval of $T$. convexa Group (3.1 Ma). The disappearance of the $T$. convexa Group described by Burckle and Trainer (1979) is not observed in this study. The T. convexa Group composes less than 1 to $5 \%$ of the diatom assemblage in samples from this interval examined at Sites 503 and 572. Samples from this interval were not examined at Site 573. The nonrecognition of the event observed by 


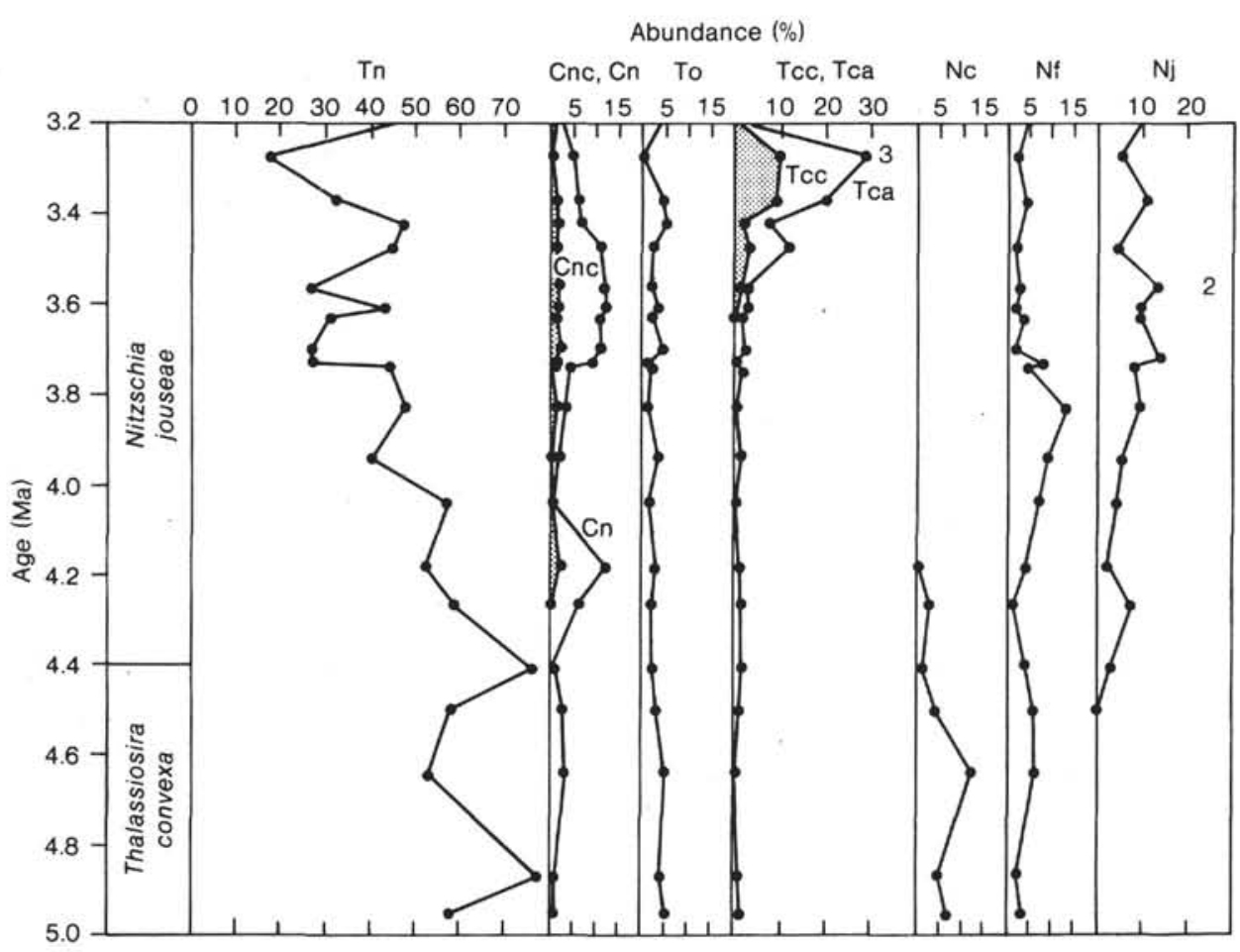

Figure 7. Fluctuations in abundance (percentage) of selected species from Site 572 for the interval 5.0 to $3.2 \mathrm{Ma}$. $\mathrm{Tn}=T$. nitzschioides; $\mathrm{Cnc}=C$. nodulifer var. cyclopus; $\mathrm{Cn}=C$. nodulifer; $\mathrm{To}=T$. oestrupii; $\mathrm{Tcc}=T$. convexa var. convexa $; \mathrm{Tca}=T$. convexa var. aspinosa $(3$, first peak in abundance of $T$. convexa Group); $\mathrm{Nc}=N$. cylindrica $; \mathrm{Nf}=N$. fossilis $; \mathrm{Nj}=N$. jouseae $(2$, acme of Actinocyclus ehrenbergii). See Tables 1 and 2 for the acme of Actinocyclus ehrenbergii. The diatom zones figured are from Burckle $(1972,1977)$. Ages of samples are extrapolated from the sediment accumulation curves (Fig. 3).

Burckle and Trainer (1979) may result from the sampling interval used during this study.

\section{Top of the Second Abundance Interval of Thalassiosira convexa Group}

The top of the second abundance interval of Thalassiosira convexa Group is also a useful datum. At the top of this second interval, $T$. convexa Group composes 10 to $20 \%$ of the overall diatom assemblage. Directly above the top of this interval, the abundance of $T$. convexa declines to $5 \%$ or less of the overall assemblage. The age of this datum is $2.97 \mathrm{Ma}$ at Site 503 and between 2.93 and $3.0 \mathrm{Ma}$ at Site 572 . Due to the sampling interval, this datum is not recognized at Site 573. An age of 2.97 Ma seems most reliable for this datum since Site 503 has four data points between 2.9 and 3.0 Ma. Although Burckle and Trainer (1979) did not recognize this datum as being useful, it does appear in their figure 1 and has an age similar to that observed in this study.

\section{Increase in the Abundance of Thalassiosira oestrupii}

At Site 573 an increase in abundance of Thalassiosira oestrupii occurs between 1.9 and $1.8 \mathrm{Ma}$ (Figs. 10 and 12). Below this interval $T$. oestrupii consistently composes less than $5 \%$ of the diatom assemblage. Above this interval T. oestrupii composes 10 to $20 \%$ of the diatom assemblage. An increase is also observed at Site
572 , but a hiatus from approximately 2.2 to $1.8 \mathrm{Ma}$ occurs there, and thus the exact placement of this increase remains a problem. The corresponding interval at Site 503 was not examined because of the poor preservation of the samples. The increase in $T$. oestrupii can only be placed tentatively within the interval approximating the first occurrence of Pseudoeunotia doliolus. A tentative age of 1.90 to $1.80 \mathrm{Ma}$ is assigned to this event. Burckle (1977) noted a second increase in abundance of $T$. oestrupii just below the Matuyama/Bruhnes boundary. This event has an approximate age of $0.75 \mathrm{Ma}$ (Burckle, 1977) and is therefore different from the event defined in this study.

Table 4 presents the datums that are considered to be stratigraphically useful and their extrapolated ages. The first occurrence of Coscinodiscus africanus, placed by Burckle (1978) within the upper part of the Gilbert Chron (Fig. 1), is not included because $C$. africanus is too sporadic after its first occurrence to be useful for stratigraphic correlation. The first occurrence of $C$. africanus may be applicable within a specific geographic province, but further studies are required.

The first occurrence of $T$. miocenica is defined by Burckle $(1972,1978)$ to occur stratigraphically below the first appearance of $T$. convexa within the upper part of paleomagnetic chron 6 . The order of appearance of these two species is reversed according to Sancetta (1982); while 


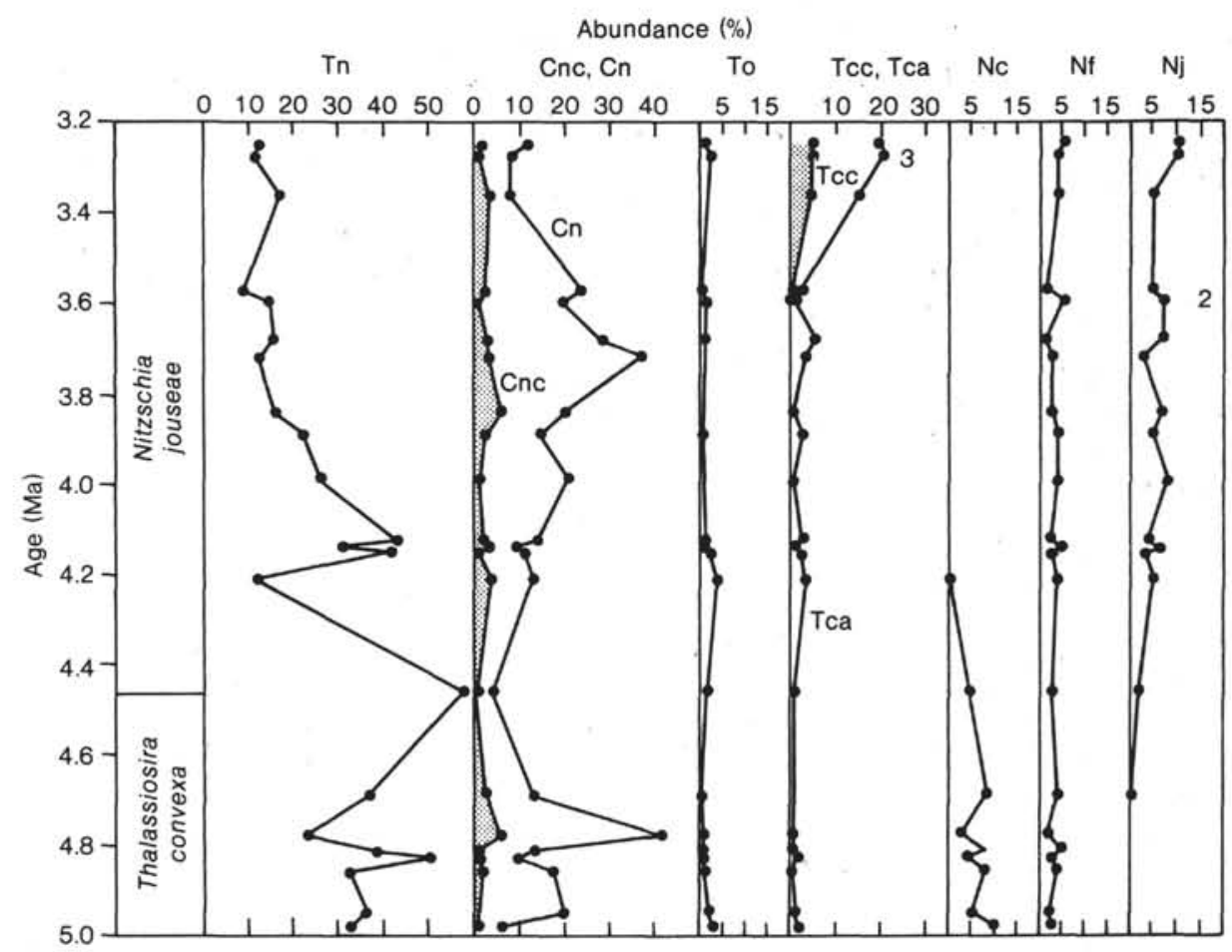

Figure 8. Fluctuations in abundance (percentage) of selected species from Site 503 for the interval 5.0 to 3.2 Ma. Abbreviations and notes same as for Figure 7.

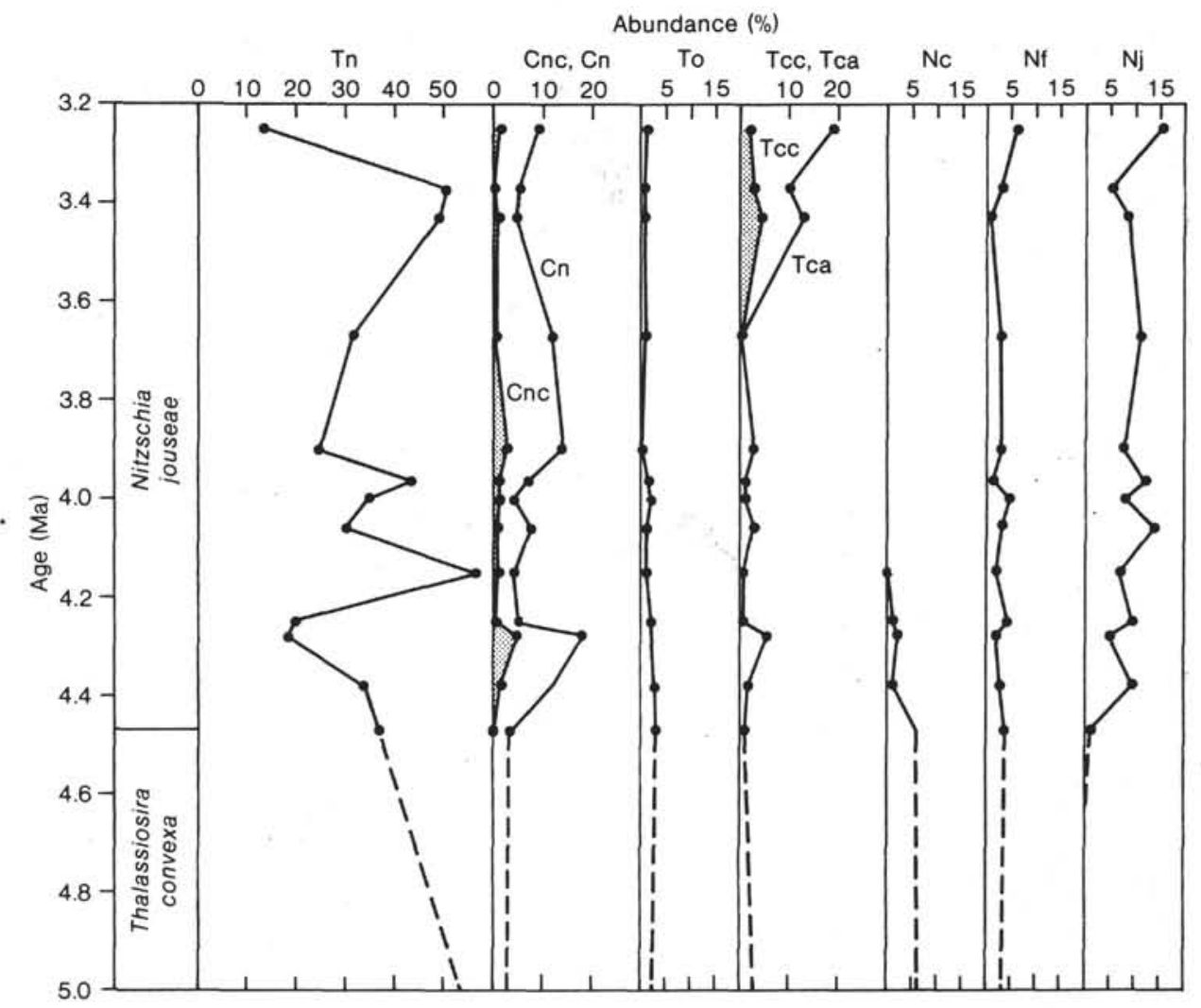

Figure 9. Fluctuations in abundance (percentage) of selected species from Site 573 for the interval 5.0 to 3.2 Ma. Dashed line represents the duration of the observed hiatus. Abbreviations and notes same as for Figure 7. 


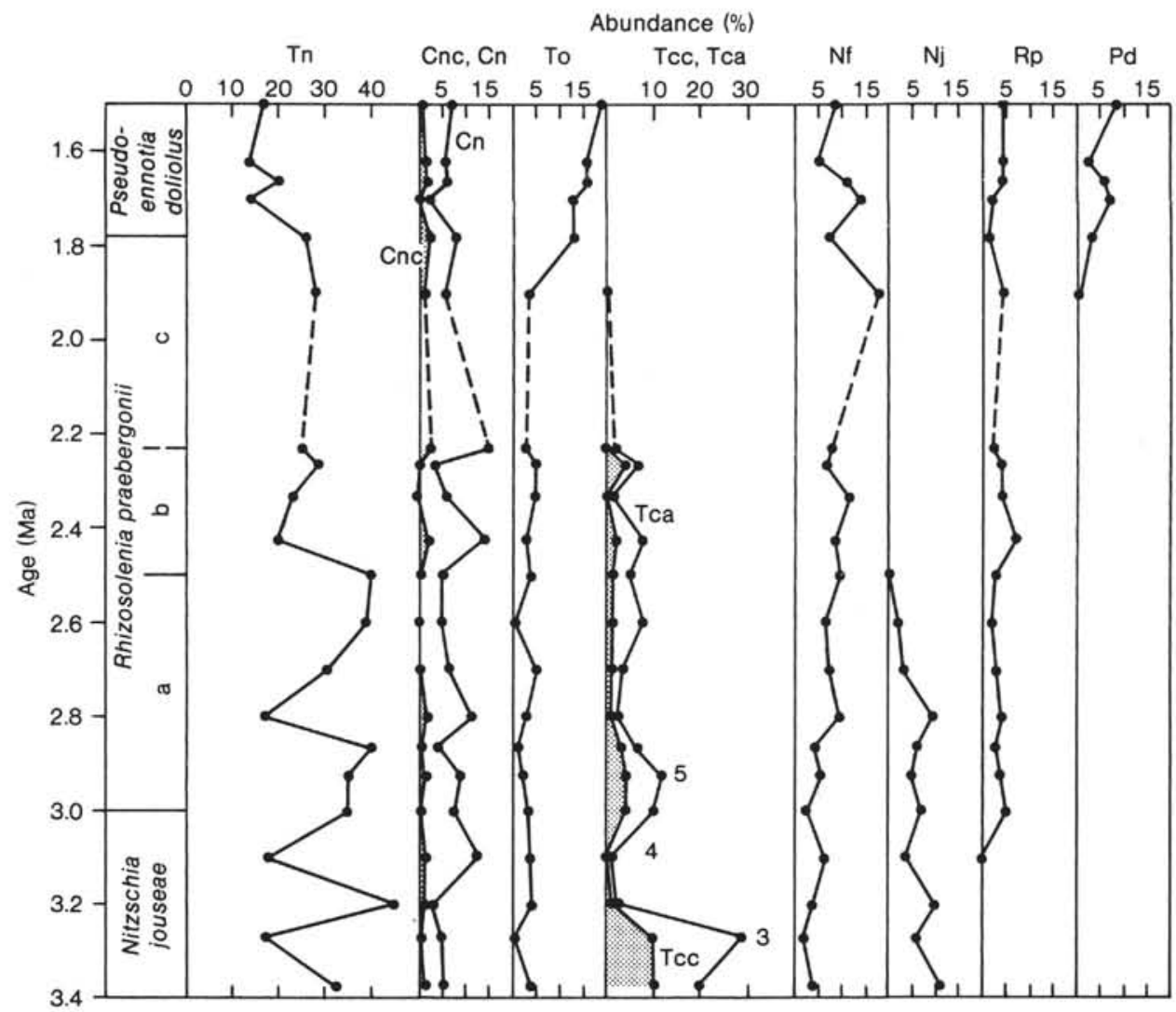

Figure 10. Fluctuations in abundance (percentage) of selected species from Site 572 for the interval 3.4 to $1.5 \mathrm{Ma} . \mathrm{Tn}=T$. nitzschioides $; \mathrm{Cnc}=C$. nodulifer var. cyclopus; $\mathrm{Cn}=C$. nodulifer $; \mathrm{To}=T$. oestrupii; $\mathrm{Tcc}=T$. convexa var. convexa $; \mathrm{Tca}=T$. convexa var. aspinosa $(3$, first peak in abundance of T. convexa Group; 4, base of second interval of abundance; 5 , second peak in abundance); $\mathrm{Nf}=N$. fossilis $; \mathrm{Nj}=N$. jouseae $; \mathrm{Rp}=R$. praebergonii $; \mathrm{Pd}=P$. doliolus. Dashed line represents the duration of the observed hiatus. Ages of samples are extrapolated from the sediment accumulation curves (Fig. 3).

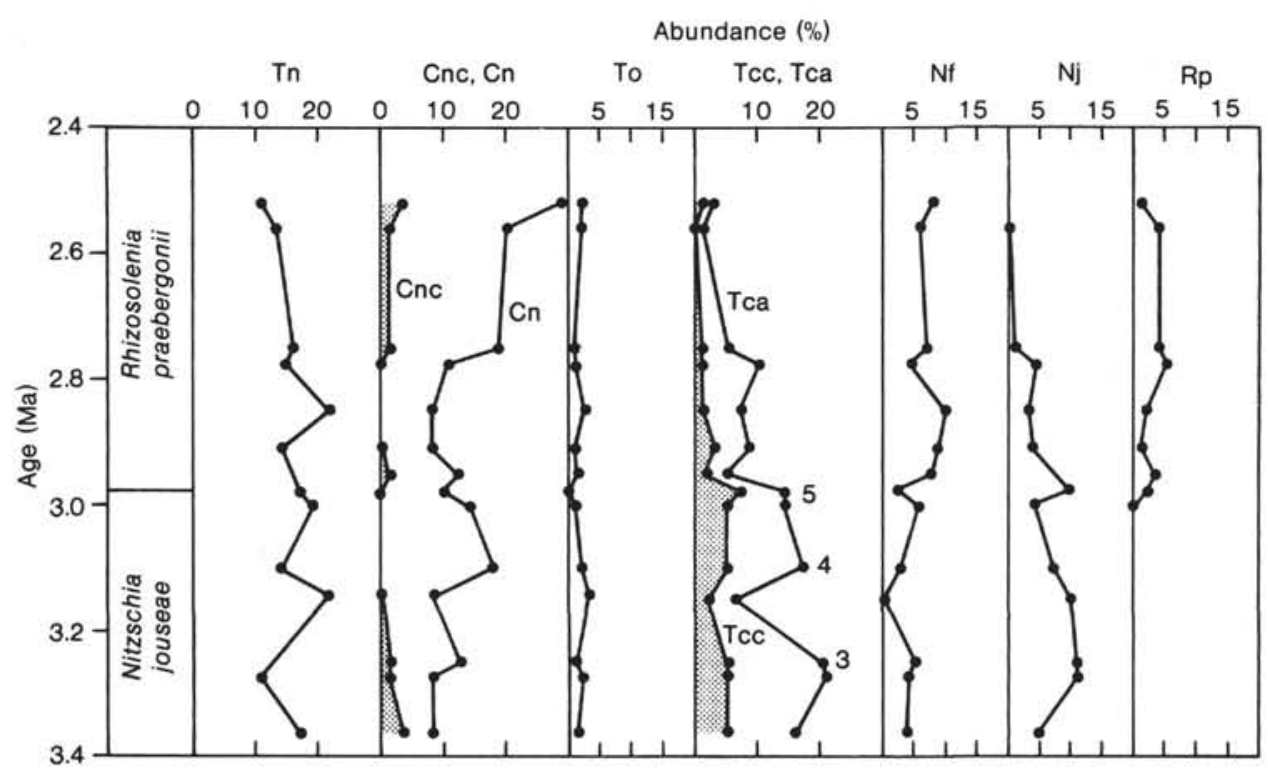

Figure 11. Fluctuations in abundance (percentage) of selected species from Site 503 for the interval 3.4 to 1.5 Ma. Abbreviations and notes same as for Figure 10. 


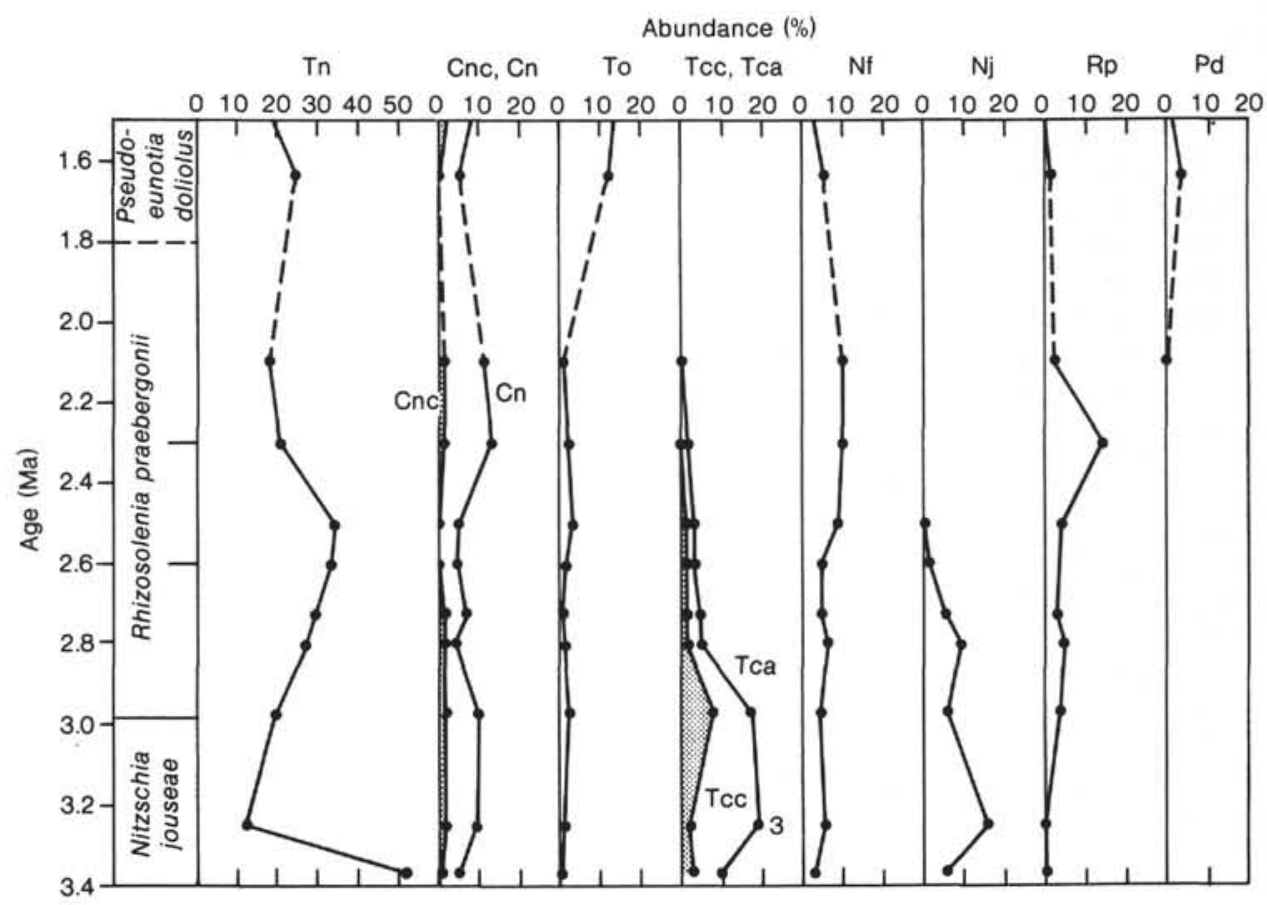

Figure 12. Fluctuations in abundance (percentage) of selected species from Site 573 for the interval 3.4 to 1.5 Ma. Dashed line represents an interval of uncertainty resulting from the sampling interval. Abbreviations and notes same as for Figure 10.

Table 4. Useful diatom datums recognized within the upper Neogene of the eastern equatorial Pacific and their extrapolated ages.

\begin{tabular}{llcl}
\hline Datum & \multicolumn{1}{c}{ Diatoms } & $\begin{array}{c}\text { Age } \\
\text { (Ma) }\end{array}$ & References \\
\hline L. & R. praebergonii var. robusta & 1.55 & $3,4,5$ \\
F. & P. doliolus & 1.80 & $1,3,4,5$ \\
L. & R. praebergonii & 1.85 & 2,3 \\
In. & T. oestrupii & $1.8-1.9$ & 6 \\
Tr. & R. praebergonii $\rightarrow$ R. praebergonii & & \\
& $\quad$ var. robusta & 2.00 & 1 \\
L. & T. convexa & 2.20 & 3 \\
F. & R. praebergonii var. robusta & 2.36 & 3 \\
L. & N. jouseae & 2.60 & 3,5 \\
Ta. & T. convexa & 2.97 & 6 \\
F. & R. praebergonii & 3.00 & 3,5 \\
Ba & T. convexa & 3.10 & 6 \\
Ta 1 & T. convexa & 3.27 & 6 \\
F. & T. convexa var. aspinosa & 3.60 & 3,5 \\
Ac. & A. ehrenbergii & $3.5-3.6$ & 6 \\
F. & A. elegans & 3.9 & 2,5 \\
L. & N. cylindrica & 4.2 & 2 \\
F. & N. jouseae & 4.5 & 2,5 \\
L. & T. miocenica & 5.1 & 2 \\
F. & T. oestrupii & $5.1-5.2$ & 6 \\
F. & N. cylindrica & 5.3 & 6 \\
L. & A. acutiloba & 5.3 & 2 \\
L. & N. miocenica & 5.5 & 2,6 \\
Ta & N. miocenica & 5.6 & 6 \\
L. & R.praepaleacea & 5.7 & 7 \\
L. & T. praeconvexa & 5.8 & 5 \\
F. & T. convexa & 6.1 & 2,5 \\
F. & T. miocenica & 6.1 & $2,4,5$ \\
F. & T. praeconvexa & 6.3 & 2 \\
\hline
\end{tabular}

Notes: 1. Burckle, 1977; 2. Burckle, 1978; 3. Burckle and Trainer, 1979; 4. Barron, 1980; 5. Barron, this volume; 6 . Baldauf, this paper; 7. Burckle, in Vincent, 1981. $\mathrm{L}=$ last occurrence; $\mathrm{F}=$ first occurrence; $\mathrm{In}=$ increase in abundance; $\mathrm{Tr}=$ evolutionary transition; $\mathrm{Ta}$ $=$ top of abundance interval; $\mathrm{Ba}=$ bottom of abundance interval; $\mathrm{Ac}=$ acme. both Baldauf (1985) and Barron (in press) recognize the first occurrences of $T$. miocenica and $T$. convexa var. aspinos $a$ as being synchronous. The difference in the stratigraphic relationship and placement of these datums probably represents differences in taxonomic concepts.

Although the last occurrence of Nitzschia cylindrica is described by Burckle (1978) as a useful secondary datum for low latitudes, no mention is made of the stratigraphic potential of this species' first occurrence. Sancetta (1982) suggests that the total stratigraphic range of $N$. cylindrica is useful, thereby suggesting that the first occurrence of this species is also useful. The present study indicates that the first occurrence of $N$. cylindrica s.s. is useful within the equatorial Pacific (Fig. 7 to 9). An age of 5.3 Ma is extrapolated for this event.

The first occurrence of $T$. oestrupii is a useful stratigraphic marker, as indicated by previous work. Burckle (1978) places the first occurrence in the equatorial $\mathrm{Pa}-$ cific slightly above the top of paleomagnetic chron 5 . Barron (1981) defined the base of his T. oestrupii Zone as the first occurrence of this taxon. Barron (1981) placed this datum within the lowermost reversed interval of the Gilbert Chron and suggested that it occurred approximately at the Miocene/Pliocene boundary. The results of the present study also indicate that the first occurrence of $T$. oestrupii approximates the Miocene/Pliocene boundary as dated by Berggren et al. (in press) (Figs. 4 to 6). An age of 5.1 to $5.2 \mathrm{Ma}$ is assigned to this first occurrence. A slight stratigraphic overlap exists between the range of $T$. oestrupii and $T$. miocenica (Figs. 4 to 6). The last occurrence of $T$. miocenica is placed at $5.1 \mathrm{Ma}$. 


\section{Calcium Carbonate Stratigraphy and Diatom Correlation}

Three factors are generally cited as responsible for fluctuations in the relative calcium carbonate content preserved within the sedimentary record. These include variation in the productivity of calcareous microplankton, dissolution of calcareous materials, and dilution by noncarbonate sediments. Unfortunately, little is understood about the mechanisms controlling these factors, although Vincent (1981) and Dunn (1982) suggest that carbonate fluctuations tend to correspond to climatic oscillations. Arrhenius (1952) originally discussed the correlation of carbonate content and climatic fluctuations. He associated sediments having a high carbonate. content with periods of cold climate and sediments having a low carbonate content with periods of warmer climate.

Although understanding the relationship between fluctuations in the carbonate content and climate oscillation is difficult, recognition of synchronous carbonate minima and maxima over a geographic region is useful in refining stratigraphic resolution. Figure 13 presents the upper Miocene to lower Pleistocene carbonate stratigraphy for Sites 572 and 573 (site chapters, this volume). The correlation of carbonate maxima and minima between sites is based on diatom datums observed at both sites.

Vincent (1981) used datums of diatom taxa in addition to those of other microfossils to correlate the Neogene carbonate stratigraphy of the Hess Rise region of the central North Pacific to the equatorial Pacific region. Comparison of the results of the present study to those of Vincent (1981) allows correlation of the carbonate stratigraphy to the diatom datums. Two distinct intervals of carbonate fluctuations can be readily correlated between Sites 572 and 573 and can be dated using diatom datums. The first interval occurs in the upper Miocene and approximates the last occurrence of Nitzschia miocenica. The second interval occurs in the upper Pliocene and corresponds to the interval between the last occurrences of Rhizosolenia praebergonii and Thalassiosira convexa.

The last occurrence of $N$. miocenica is at a depth of $140.8 \mathrm{~m}$ at Site 572 and $95.0 \mathrm{~m}$ at Site 573 . This datum correlates at both sites to the transition between the carbonate maximum $5 \mathrm{~d}$ and the carbonate minimum $5 \mathrm{e}$ (Fig. 13). Directly below the last occurrence of $N$. miocenica is the peak in abundance of $N$. miocenica that correlates to the transition between the carbonate minimum $5 \mathrm{e}$ and carbonate maximum $5 \mathrm{f}$. Vincent (1981) correlates the carbonate event $5 \mathrm{~d}$ with the last occurrence of $N$. miocenica in Core RC12-66 in the central North Pacific Ocean. The slight difference between the correlation by Vincent (1981) and this study (approximately $50,000 \mathrm{yr}$.) is the probable result of sampling differences and biostratigraphic resolution.

The upper Pliocene sequence, within which 3 diatom datums occur (first $R$. praebergonii; last $N$. jouseae; and last $T$. convexa), is easily correlated between Sites 572 and 573 and occurs from 47 to $35 \mathrm{~m}$ at Site 572 and from 43 to $33 \mathrm{~m}$ at Site 573 . These datums allow the cor-

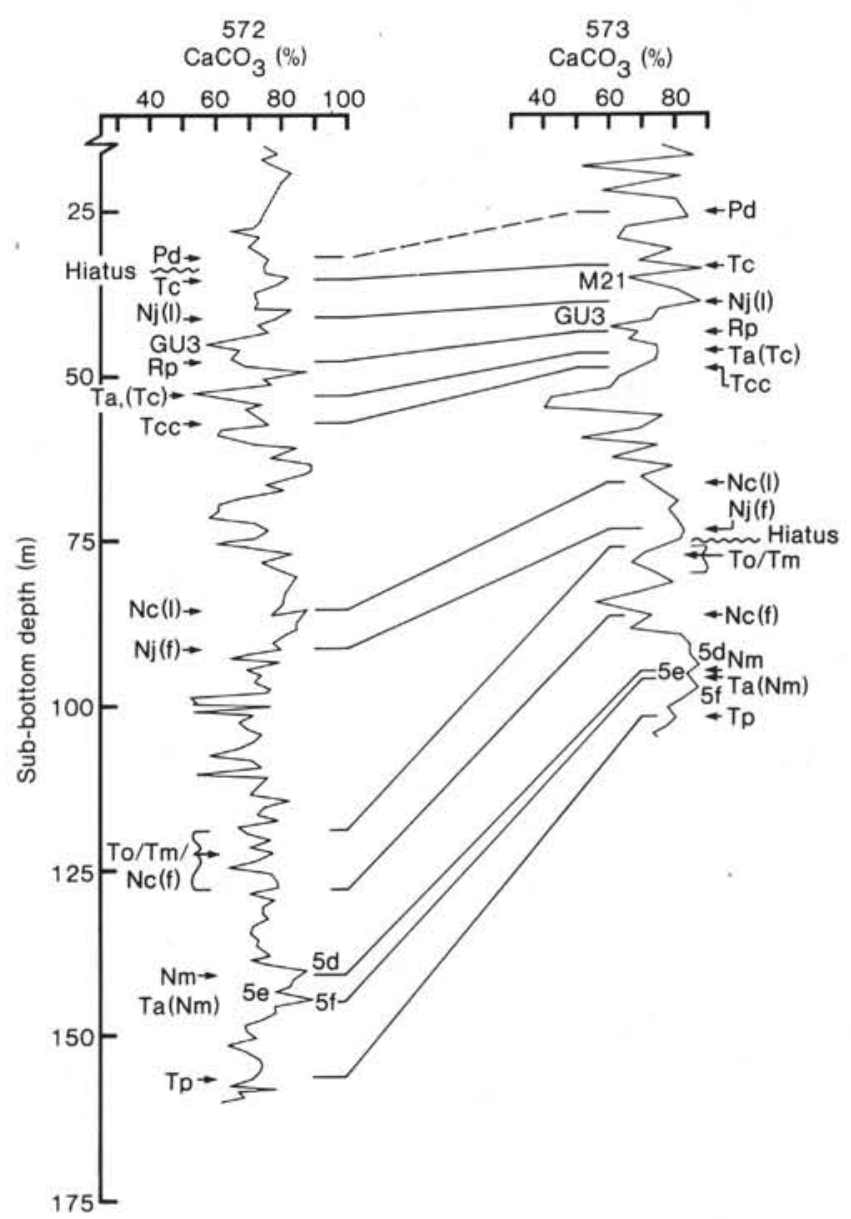

Figure 13. Correlation of the carbonate stratigraphy from Sites 572 and 573 (site chapters, this volume) to the late Neogene diatom datums observed at both sites. The carbonate peaks are labeled following Vincent $(1981)$. $\mathrm{Tp}=$ last $T$. praeconvexa; $\mathrm{Ta}(\mathrm{Nm})$ peak of abundance of $N$. miocenica; $\mathrm{Ta}(\mathrm{Tc})$ peak of abundance of $T$. miocenica $; \mathrm{Nm}=$ last $N$. miocenica $;$ To $=$ first $T$. oestrupii; $\mathrm{Tm}=$ last $T$. miocenica; $\mathrm{Nc}(\mathrm{f})=$ first $N$. cylindrica; $\mathrm{Nj}(\mathrm{f})=$ first $N$. jouseae $\mathrm{Nc}(\mathrm{l})=$ last $N$. cylindrica; $\mathrm{Tcc}=$ first $T$. convexa var. convexa $; \mathrm{Rp}=$ first $R$. praebergonii; $\mathrm{Nj}(\mathrm{l})=$ last $N$. jouseae; $\mathrm{Tc}$ $=$ last $T$. convexa $; \mathrm{Pd}=$ first $P$. doliolus .

relation of the carbonate minima Gu3 and M21 between these sites (Fig. 13).

Carbonate minimum Gu3 occurs at $45 \mathrm{~m}$ depth at Site 572 and at $42 \mathrm{~m}$ at Site 573 , directly above the first occurrence of $R$. praebergonii at $47.5 \mathrm{~m}$ at Site 572 and $43 \mathrm{~m}$ at Site 573. Vincent (1981) correlates the Gu3 carbonate minimum at Site RC12-66 and DSDP Site 157 in the central North Pacific with the first occurrence of $R$. praebergonii var. $A$. However, it is clear from the rest of her data that Gu3 coincides with the first occurrence of $R$. praebergonii, which is in agreement with observations at Sites 572 and 573.

Other carbonate peaks shown in Figure 13 are not discussed here because of the uncertainty of their correlation. The use of only diatom datums for correlation of these carbonate peaks results in this uncertainty.

\section{Diatom Abundance Stratigraphy}

Coscinodiscus nodulifer has a greater average abundance at Site 503 than at Sites 573 and 572 (Figs. 4-12). 
At Site 503 C. nodulifer composes an average of $15 \%$ of the total diatom assemblage. Occasional peaks of 30 to $40 \%$ are also observed. At Site 573 the average abundance of $C$. nodulifer is slightly less, having an average of $10 \%$. The occasional peaks observed for this site are also less and have a maximum value of $20 \%$. The lowest average abundance of $C$. nodulifer occurs at Site 572, where values of $5 \%$ are common. The abundance peaks at this site are further reduced, with a maximum value of $15 \%$. The abundance of $C$. nodulifer at Site 572 may be influenced not only by the effects of equatorial upwelling but also by the Peru-Chile Current. This is suggested by the abundance of Thalassionema nitzschioides at Site 572 .

Site 503 is geographically situated within the Guatemala Basin, which is a region characterized by equatorial upwelling. The greater abundance of $C$. nodulifer at Site 503 suggests that $C$. nodulifer favors equatorial upwelling conditions. A similar conclusion was reached by Cooke-Poferl et al. (1975) on the basis of three diatom assemblages that were recognized from 180 core tops in the eastern equatorial Pacific region. They recognized an assemblage dominated by $C$. nodulifer that was associated with equatorial upwelling. The additional assemblages recognized by Cooke-Poferl et al. (1975) include an assemblage dominated by $T$. nitzschioides (associated with the Peru-Chile Current) and an assemblage dominated by Pseudoeunotia doliolus and T. oestrupii (associated with the North Equatorial Counter Current).

The fluctuations in the relative frequency of $T$. nitzschioides also merit discussion. T. nitzschioides has the greatest average abundance at Site 572, where it averages $40 \%$ of the total diatom assemblage (see Fig. 14). At Sites 573 and 503 the abundance of $T$. nitzschioides is slightly less, averaging 36 and $32 \%$, respectively. Previous studies have associated $T$. nitzschioides with coastal upwelling (Schuette and Schrader, 1979). In addition, Cooke-Poferl et al. (1975) associate the dominance of $T$. nitzschioides in the eastern equatorial Pacific with the northward-flowing Peru-Chile Current. This study suggests that $T$. nitzschioides is also associated with the upwelling of the Equatorial Undercurrent.

Site 572 , at the western edge of the productivity region, has the greatest abundance of $T$. nitzschioides, which can be divided into three intervals (Fig. 14). The first interval extends from 6.1 to $\sim 4.2 \mathrm{Ma}$. Within this interval, $T$. nitzschioides composes 50 to $65 \%$ of the total diatom assemblage. Between 4.2 and $3.8 \mathrm{Ma}$ the abundance of $T$. nitzschioides declines from 65 to $40 \%$. Above this interval $T$. nitzschioides composes between 15 and $40 \%$ of the assemblage.

Although the average abundance of $T$. nitzschioides is less at Site 503, a trend in abundance similar to that at Site 572 is observed within the high productivity region (Fig. 14). Between 6.1 and $\sim 4.1 \mathrm{Ma}, T$. nitzschioides composes from 30 to $55 \%$ of the assemblage. A gradual decline in abundance from 30 to $15 \%$ is observed between 4.1 and $3.9 \mathrm{Ma}$. Stratigraphically above this decline, $T$. nitzschioides composes from 10 to $20 \%$ of the diatom assemblage (Fig. 14).
The trend in abundance is not as pronounced at Site 573 in the low productivity region (Fig. 14). The abundance of $T$. nitzschioides at Site 573 ranges from 20 to $65 \%$. A distinct pulse of T. nitzschioides (from 40 to $65 \%$ ) is observed at $5.4 \mathrm{Ma}$. Although at Site $573 \mathrm{~T}$. nitzschioides shows a slight decline near the top of the examined interval, a decline in abundance similar to that at Sites 503 and 572 between 4.2 and $3.7 \mathrm{Ma}$ is not observed. The gradual decline in abundance of T. nitzschioides at both Sites 572 and 503 in the easternmost Pacific may represent a change in oceanographic circulation between 4.2 and $3.7 \mathrm{Ma}$. The absence of a similar decline at Site 573 suggests that the effects of this oceanographic change (if responsible) would have been restricted, or at least most extreme, within the easternmost equatorial Pacific.

Prior to the closing of the Isthmus of Panama, $T$. nitzschioides may have been associated with not only the Peru-Chile Current but also with the zone of mixing that may have resulted from the influx and interaction of warm, high-salinity Atlantic surface water with cooler, lower salinity Equatorial Undercurrent water. If this hypothesis is correct, T. nitzschioides would have had a greater abundance in the region of Sites 572 and 503 while communication between the two oceans remained open. Once this communication was severed (as a result of the emergence of the Isthmus of Panama), water masses within the easternmost equatorial Pacific would have had to reorganize. In particular, the geographic location of the upwelling of the eastern Equatorial Undercurrent would have been affected. This would presumably have resulted in conditions less favorable to $T$. nitzschioides and thus in a decline in its abundance. Although the above model presents a reasonable explanation for the fluctuation in the abundance of $T$. nitzschioides, further evidence should be evaluated.

\section{CONCLUSION}

The stratigraphic resolution for the upper Neogene of the eastern equatorial Pacific is refined by the recognition of six new diatom events. Recognition of these datums is the result of quantitative methods. In addition, stratigraphic resolution is improved in the equatorial Pacific by the correlation of diatom events to the carbonate events 5f, 5e, 5d, Gu3, and M21.

The fluctuations in abundance revealed by quantitative methods suggest changes in paleoceanographic circulation. The decline in abundance of $T$. nitzschioides between 4.2 and $3.7 \mathrm{Ma}$ in the easternmost sites may be related to the restriction in communication between the Atlantic and Pacific resulting from emergence of the Isthmus of Panama. The absence of a decline in T. nitzschioides at the more westerly Site 573 during the same time interval suggests that the easternmost Pacific was most affected by this closure. Fluctuations in abundances of species also allow inferences to be made about the association of species to ecological conditions. The association of $C$. nodulifer with equatorial upwelling and T. nitzschioides with the Peru-Chile Current and 


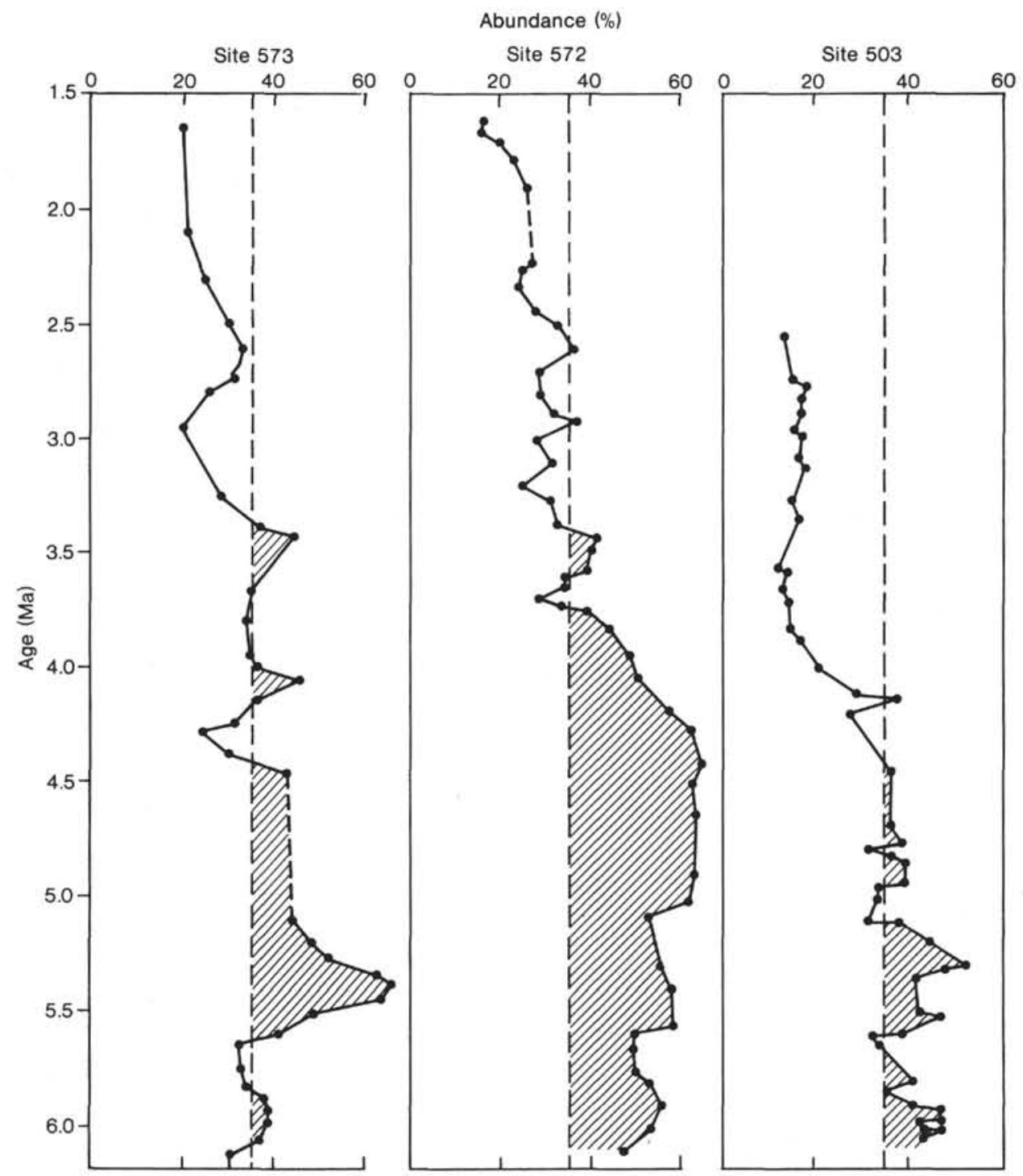

Figure 14. Correlation of the fluctuations in abundance (percentage) of T. nitzschioides for Sites 573, 572, and 503. The ages of the samples are extrapolated from the sediment accumulation curves (Fig. 3). Vertical dashed lines are a frame of reference to show the decline in abundance at Sites 572 and 503 between 4.2 and $3.7 \mathrm{Ma}$.

the Equatorial Undercurrent suggest changes in circulation related to the uplift of the Isthmus of Panama.

\section{ACKNOWLEDGMENTS}

I wish to thank John Barron, Charles Blome, Charlotte Brunner, Dave Bukry, and Charles Repenning for reviewing this manuscript. In addition, I would like to express my thanks to John Barron for his helpful discussion during this study. Appreciation is further due to John Barron, James Gardner, and the National Science Foundation for providing the samples examined.

\section{REFERENCES}

Arrhenius, G., 1952. Sediment cores from the east Pacific. Rep. Swed. Deep Sea Exped., 1947-1948, 5:1-202.

Baldauf, J. G., 1985. Cenozoic diatom biostratigraphy and paleoceanography of the Rockall Plateau Region, North Atlantic, Deep Sea Drilling Project Leg 81. In Roberts, D., Schnitker, D., et al., Init.
Repts. DSDP, 81: Washington (U.S. Govt. Printing Office), 439-478.

Barron, J. A., 1980. Upper Pliocene and Quaternary diatom biostratigraphy of the Deep Sea Drilling Project Leg 54. In Rosendahl, B. R., Hekinian, R., et al., Init. Repts. DSDP, 54: Washington (U.S. Govt. Printing Office), 455-485.

1981. Late Cenozoic diatom biostratigraphy and paleoceanography of the middle latitude eastern North Pacific, Deep Sea Drilling Project Leg 63. In Yeats, R. S., Haq, B. U., et al., Init. Repts. DSDP, 63: Washington (U.S. Govt. Printing Office), 507-538.

in press. Neogene planktic diatom biostratigraphy. In Saunders, J. B., and Bolli, H. M., (Eds.), Biostratigraphy by Marine Plankton: Cambridge (Cambridge Univ. Press).

Barron, J. A., and Keller, G., 1982. Widespread Miocene deep-sea hiatuses: Coincidence with periods of global cooling. Geology, 10: 577-581.

Barron, J. A., Keller, G., Dunn, D. A., Kennett, J. P., Lombari, G., Burckle, L. H., and Vinvent, E., in press. A multiple microfossil 
biochronology for the Miocene. In Savin, S. M. (Ed.), Cenozoic Paleoceanographic Synthesis (CENOP), Geol. Soc. Am. Mem.

Berggren, W. A., Kent, D. V., and Flynn, J. J., in press. Neogene geochronology and chronostratigraphy. Geochronology and the Geological Record, Geol. Soc. London Spec. Pap.

Burckle, L. H., 1969. Late Cenozoic planktonic diatom zones in equatorial Pacific sediments. Geol. Soc. Am. Abstr. Progr., 7:24. (Abstract)

1972. Late Cenozoic planktic diatom zones from the eastern equatorial Pacific. Nova Hedwigia, 39:150-217.

1977. Pliocene and Pleistocene diatom datum levels from the equatorial Pacific. Quat. Res. (NY), 7:330-340.

, 1978. Early Miocene to Pliocene diatom datum levels for the equatorial Pacific. Proc. Second Working Group Mtg., Biostratigraphic Datum Planes of the Pacific Neogene, IGCP Project 114: Bandung, Indonesia (Geol. Res. Dev. Ctr.), 1:25-44.

Burckle, L. H., and Trainer, J., 1979. Middle Miocene and late Pliocene diatom datum levels from the Central Pacific. Micropaleontology, 25(3):281-293.

Cooke-Poferl, K., Burckle, L. H., and Riley, S., 1975. Diatom evidence bearing on late Pleistocene climatic changes in the equatorial Pacific. Geol. Soc. Am. Ann. Meet., 1038-1039.

Dunn, D. A., 1982. Changes from "Atlantic-type" to "Pacific-type" carbonate stratigraphy in middle Pliocene equatorial Pacific ocean. Mar. Geol., 50:41-60.
Hailwood, E. A., and Rees, A. I., 1982. Magnetic properties of sediments from Hole 503A. In Prell, W. L., Gardner, J. V., et al., Init. Repts. DSDP, 68: Washington (U.S. Govt. Printing Office), 441-444.

Keller, G., and Barron, J. A., 1983. Paleoceanographic implications of Miocene deep-sea hiatuses. Geol. Soc. Am. Bull., 94:590-613.

Sancetta, C., 1982. Diatom biostratigraphy and paleoceanography, Deep Sea Drilling Project Leg 68. In Prell, W. L., Gardner, J. V., et al., Init. Repts. DSDP, 68: Washington (U.S. Govt. Printing Office), 301-310.

Schrader, H. J., and Gersonde, R., 1978. Diatoms and silicoflagellates. Utrect Micropaleonotol. Bull., 17:129-176.

Schuette, G., and Schrader, H. J., 1979. Diatom taphocenoses in the coastal upwelling area off western South America. Nova Hedwigia, 64:359-378.

Vincent, E., 1981. Neogene carbonate stratigraphy of the Hess Rise (central north Pacific) and paleoceanographic implications. In Thiede, J., Vallier, T. L., et al., Init. Repts. DSDP, 62: Washington (U.S. Govt. Printing Office), 571-606.

Date of Initial Receipt: 10 October 1983

Date of Acceptance: 28 February 1984 